\title{
The Absolute Magnitude of the Sun in Several Filters
}

\author{
Christopher N. A. Willmer (i) \\ Steward Observatory, University of Arizona 933 North Cherry Avenue Tucson, AZ 85721, USA; cnaw@ as.arizona.edu \\ Received 2018 April 4; revised 2018 April 18; accepted 2018 April 19; published 2018 June 14
}

\begin{abstract}
This paper presents a table with estimates of the absolute magnitude of the Sun and the conversions from vegamag to the $\mathrm{AB}$ and $\mathrm{ST}$ systems for several wide-band filters used in ground-based and space-based observatories. These estimates use the dustless spectral energy distribution (SED) of Vega, calibrated absolutely using the SED of Sirius, to set the vegamag zero-points and a composite spectrum of the Sun that coadds space-based observations from the ultraviolet to the near-infrared with models of the Solar atmosphere. The uncertainty of the absolute magnitudes is estimated by comparing the synthetic colors with photometric measurements of solar analogs and is found to be $\sim 0.02 \mathrm{mag}$. Combined with the uncertainty of $\sim 2 \%$ in the calibration of the Vega SED, the errors of these absolute magnitudes are $~ 3 \%-4 \%$. Using these SEDs, for three of the most utilized filters in extragalactic work the estimated absolute magnitudes of the Sun are $M_{B}=5.44$, $M_{V}=4.81$, and $M_{K}=3.27 \mathrm{mag}$ in the vegamag system and $M_{B}=5.31, M_{V}=4.80$, and $M_{K}=5.08 \mathrm{mag}$ in $\mathrm{AB}$.
\end{abstract}

Key words: astronomical databases: miscellaneous - catalogs

Supporting material: data behind figure

\section{Introduction}

Several astrophysical quantities, such as the masses and luminosities of stars and galaxies, are often described in terms of solar units. The luminosity density (the integral of the luminosity function) is even more specific, as it is usually expressed in terms of solar luminosities within a given photometric band (e.g., $B$ or $K$ ). The consistent absolute calibration of flux measurements is still an essential endeavor in astrophysics, because of the expansion of wavelength coverage and the ever increasing sensitivity of instruments both from the ground and space (see Bohlin et al. 2014 for a comprehensive review). Because the first catalogs of stellar photometry used Vega as the prime calibrator (Johnson \& Morgan 1953; Johnson 1955, 1966), magnitudes are commonly referred to that star. However, to overcome the effects of dust and molecular lines on stellar spectra which are difficult to model, there has been a shift to adopt either the AB system of Oke \& Gunn (1983), where the calibrating spectrum is flat in $f_{\nu}$, or the ST system (Bessell et al. 1998; Space Telescope Science Institute 1998), for a flat spectrum in $f_{\lambda}$. Both, in their turn, can be referred to observations of white dwarfs, which are calibrated through stellar models and ultimately through the use of laboratory reference standards (Bohlin et al. 2014).

Previous compilations of the Sun's absolute magnitude were published by Binney \& Merrifield (1998) for the Johnson-Cousins-Glass system and Blanton et al. (2003) for the Sloan Digital Sky Survey (SDSS) filters redshifted to $z=0.1$ in AB magnitudes. Engelke et al. (2010) calculated the apparent magnitude of the Sun for several filters, including the Johnson-Cousins (UBVRI), 2MASS (JHK), and Spitzer IRAC $8 \mu \mathrm{m}$ and MIPS $24 \mu \mathrm{m}$, which can be easily converted into absolute magnitudes. The conversion constants between the vegamag system, where the absolute calibration is referred to Vega and the $\mathrm{AB}$ (Oke \& Gunn 1983) and ST (Bessell et al. 1998; Space Telescope Science Institute 1998) systems for different filters, are less easy to find, and the most extensive compilation of the vegamag to $\mathrm{AB}$ measurements was published by Fukugita et al. (1995). The aim of this paper is to provide a handy reference for the absolute magnitude of the Sun in several filters used primarily by large surveys, and the additive constants (i.e., the magnitude of Vega) that transform vegamag into the $\mathrm{AB}$ and $\mathrm{ST}$ systems. This is done using recent determinations of the spectral energy distribution (SED) of Vega and the Sun derived from space-based observations combined with models of the atmospheres of these stars.

This paper is organized as follows. Section 2 describes the filter curves, the measurement of synthetic magnitudes, and the determination of the vegamag zero-points; Section 3 describes the construction of the solar spectrum; Section 4 contains a summary and conclusions.

\section{Filter Curves and Synthetic Magnitudes}

The filter profiles were compiled from the literature, e.g., Tonry et al. (2012), Mann \& von Braun (2015), or downloaded from the databases of observatories or surveys, e.g., JWST, Dark Energy Survey. The filter profiles include the throughput due to the telescope, instrument optics, and detector quantum efficiency (e.g., $H S T$ and JWST filters). For $H S T$ filters, the latest files available in the synphot database ${ }^{1}$ were used. Most of the filters used in the ground-based observations, e.g., SDSS (Gunn et al. 1998) and Pan-STARRS (Tonry et al. 2012), also include a contribution due to the Earth's atmosphere. As the JWST Mid Infra Red Instrument (MIRI) filter response curves of Glasse \& MIRI European Consortium (2015) only contain the instrument throughput, these were multiplied by the expected JWST mirror reflectance as provided by the STScI NIRCam Team.

The reconstruction of the full system throughput using CCD photometry for the $U, B, V$ bands of Johnson \& Morgan (1953)

\footnotetext{
http://www.stsci.edu/hst/observatory/crds/throughput.html
} 
Table 1

Template Colors

\begin{tabular}{|c|c|c|c|c|c|c|}
\hline $\begin{array}{l}\text { Color } \\
\text { (1) }\end{array}$ & $\begin{array}{l}\text { Sirius }^{\mathrm{a}} \\
\text { (2) }\end{array}$ & $\begin{array}{c}\text { Bessell et al. (1998) } \\
\text { (3) }\end{array}$ & $\begin{array}{c}\mathrm{BD}+601753^{\mathrm{b}} \\
(4)\end{array}$ & $\begin{array}{l}\operatorname{IRSA}^{\mathrm{c}} \\
\quad(5)\end{array}$ & $\begin{array}{c}\text { Rieke et al. (2008) } \\
\text { (6) }\end{array}$ & $\begin{array}{c}\text { Engelke et al. (2010) } \\
\text { (7) }\end{array}$ \\
\hline$U-B$ & -0.054 & -0.045 & 0.008 & $\ldots$ & 0.022 & -0.029 \\
\hline$B-V$ & -0.015 & -0.01 & $0.023^{\mathrm{d}}$ & $0.080^{\mathrm{d}, \mathrm{e}}$ & 0.001 & -0.005 \\
\hline$V-R$ & -0.013 & -0.012 & 0.007 & $\cdots$ & -0.006 & 0.010 \\
\hline$R-I$ & -0.016 & -0.008 & 0.009 & $\ldots$ & -0.005 & 0.011 \\
\hline$V$-2MASS_K & -0.089 & $-0.061^{\mathrm{f}}$ & 0.028 & $\ldots$ & -0.028 & -0.025 \\
\hline 2MASS_J-2MASS_H & -0.015 & $-0.018^{f}$ & 0.003 & -0.039 & -0.004 & 0.008 \\
\hline 2MASS_H-2MASS_Ks & -0.006 & $-0.009^{f}$ & 0.002 & 0.006 & -0.003 & -0.019 \\
\hline IRAC_3.6-IRAC_4.5 & -0.002 & $\cdots$ & -0.000 & 0.013 & 0.001 & -0.003 \\
\hline IRAC_4.5-IRAC_5.8 & -0.002 & $\cdots$ & -0.000 & -0.006 & -0.000 & -0.005 \\
\hline IRAC_5.8-IRAC_8.0 & -0.003 & $\cdots$ & -0.002 & -0.010 & -0.001 & -0.001 \\
\hline WISE_1-WISE_2 & -0.003 & $\ldots$ & -0.000 & -0.027 & 0.000 & -0.005 \\
\hline WISE_2-WISE_3 & -0.008 & $\ldots$ & -0.005 & -0.025 & -0.001 & -0.002 \\
\hline WISE_3-WISE_4 & -0.006 & $\cdots$ & -0.004 & $0.596^{\mathrm{g}}$ & 0.000 & 0.010 \\
\hline
\end{tabular}

Notes.

a sirius_stis_002.fits.

b NASA/IPAC Infrared Science Archive (2008) archive.

c bd60d1753_stis_004.fits.

d Tycho filters (Høg et al. 2000).

e Høg et al. (2000).

${ }^{\mathrm{f}}$ Carter (1990) SAAO system.

${ }^{g}$ Low S/N measurement in WISE_4.

and Johnson (1955), and $R$ and $I$ of Cousins (1976), which were measured using photoelectric photometers, has been addressed in several works, among which are Maíz Apellániz (2006), Bessell \& Murphy (2012, hereafter BM12), and Mann $\&$ von Braun (2015). In the latter work, the authors redetermine the profiles of 39 filters $(U, B, V, R$, and $I$ among them) using spectroscopic libraries from the HST/STIS and IRTF/SPEX instruments, which provide coverage from the ultraviolet (UV) shortward of the atmospheric cutoff to the near-infrared. In their comparison with BM12, Mann \& von Braun (2015) found agreement within $2 \%$ for most filters, a notable exception being $U$, which shows a $5 \%$ difference, which they traced to the use by BM12 of the MILES library (Falcón-Barroso et al. 2011), which has less extensive $U$ coverage than the STIS spectroscopy used by Mann \& von Braun (2015).

The wavelength limits of filter curves adopted in this work are set by the wavelengths where the system throughput reaches below $10^{-4}$ of the peak value. The filters are normalized by the maximum value and then resampled using linear interpolation because using spline interpolations can introduce spurious features in filters that do not have smooth curves (e.g., 2MASS).

The calculation of synthetic magnitudes follows BM12's Equation (A11):

$$
\text { magnitude }=-2.5 \log _{10}\left[\frac{\int f_{\lambda}(\lambda) R(\lambda) \lambda d \lambda}{\int R(\lambda) \lambda d \lambda}\right]-z p,
$$

where $f_{\lambda}(\lambda)$ is the stellar flux density in erg $\mathrm{cm}^{-2} \mathrm{~s}^{-1} \AA^{-1}$, $R(\lambda)$ is the product of the detector quantum efficiency $\times$ filter throughput $\times$ unitless fractional transmission of the total telescope optical train, and $z p$ is the zero-point correction for a given magnitude system. The integral is calculated at each filter wavelength by determining the stellar flux value using linear interpolation.

The $A B$ system is defined such that the zero-point flux density for every filter is $3631 \mathrm{Jy}$, corresponding to a $z p=+48.6$ For the ST system the $z p=+21.1$ and is defined such that the magnitude of Vega in the (Johnson) $V$ band is +0.03 (Bessell et al. 1998). In both cases these zero-points assume the standard calibration spectrum is flat either in frequency $(\mathrm{AB})$ or wavelength $(\mathrm{ST})$ (Space Telescope Science Institute 1998).

In the case of the Johnson (1966) UBVRI or vegamag (Space Telescope Science Institute 1998) system, the zero-point is defined from the colors of several A stars, and because of this, Vega has a small magnitude offset in all bands that must be accounted for when using its spectrum as a flux standard (Rieke et al. 2008). However, the finding that Vega's spectrum shows the presence of a debris disk (Aumann et al. 1984; Rieke et al. 2008; Su et al. 2013; Bohlin 2014), and that in addition is a rapid rotator (Peterson et al. 2006), limits the ability of theoretical models of matching its SED, and has prompted the search of other AV stars to serve as spectral flux standards, e.g., Cohen et al. (1992), Bessell et al. (1998), Engelke et al. (2010), Bohlin (2014).

The use of Sirius as a flux standard for the infrared was initially proposed by Cohen et al. (1992), and adopted by Bessell et al. (1998) and Engelke et al. (2010). A detailed analysis of the SED of Sirius was done by Bohlin (2014) who created a template combining IUE and HST/STIS spectra for wavelengths between $\sim 0.15$ and $1.0 \mu \mathrm{m}$ with a Kurucz model of Sirius to $300 \mu \mathrm{m}$. Bohlin (2014) found the STIS measurements to agree to better than $1 \%$ with the Kurucz model and that this model also shows good agreement ( $2 \%$ ) with infrared photometry obtained by the Midcourse Space Experiment $(M S X)$ satellite. Based on these results, Bohlin (2014) concluded that Sirius can be used as a standard 


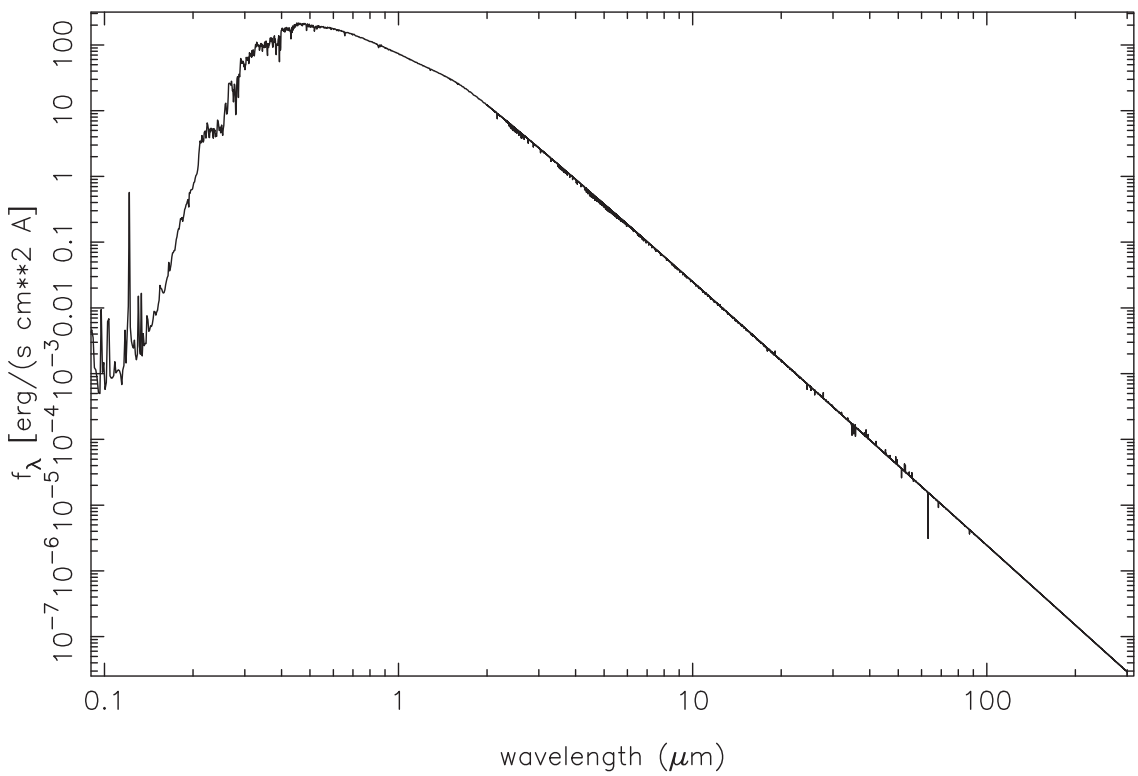

Figure 1. Composite spectrum of the Sun, combining the observed spectrum of Haberreiter et al. (2017) to $2.0 \mu \mathrm{m}$, the Fontenla et al. (2011) model between 2.0 and $100 \mu \mathrm{m}$ and the Kurucz model Sun_mod_001.fits from 100 to $300 \mu \mathrm{m}$. The data used to create this figure are available.

calibrator for the infrared and its composite spectrum is available in the CALSPEC database (sirius_stis_002.fits). After adopting Sirius as the flux standard, Bohlin (2014) renormalized the Vega composite dust-free template spectrum that combines IUE and STIS observations of Vega with two Kurucz models for Vega with $T=9550 \mathrm{~K}$ (for the extreme $\mathrm{UV}$ ) and $T=9400 \mathrm{~K}$ (for the visible-far-IR) (Bohlin 2014), which is file alpha_lyr_stis_008.fits in the CALSPEC database.

Other AV templates have been defined using models and observations. Rieke et al. (2008) constructed a dustless A0V template using the Kurucz (2005) model of Vega and normalized the spectrum in the infrared after correcting for the contribution of the debris disk. By means of a detailed comparison with the photometry of A dwarfs and solar analogs Rieke et al. (2008) showed that this A0V template, as well as the solar SED they calculated in the same paper, give consistent calibrations for the infrared. An AV template combining ground-based observations of 109 Vir with the average NICMOS observations for eight A type stars, ISO observations of Sirius (from 2.4 to $9.4 \mu \mathrm{m}$ ), and beyond $9.4 \mu \mathrm{m}$, a Kurucz model spectrum for Sirius, was compiled by Engelke et al. (2010), who found that the calibration uncertainties are $\lesssim 2 \%$. The final template considered here is the A1V star BD+60 1753, which is one of the IRAC calibrators (Reach et al. 2005) that has a CALSPEC spectrum bd60d1753_stis_004.fits, which combines HST/STIS observations from 1140 to $10120 \AA$ with BOSZ models beyond $10120 \AA$ (Bohlin et al. 2017).

A comparison between colors measured using the Bohlin (2014) alpha_lyr_stis_008.fits spectrum of Vega as a standard (which will be zero by definition) with those of the AV templates discussed above is shown Table 1. Column (1) is the photometric color, column (2) is the synthetic color measured using the Sirius spectrum of Bohlin (2014), and column (3) is photometric measurement of Sirius in Bessell et al. (1998). Column (4) shows the synthetic photometry colors for $\mathrm{BD}+60$ 1753, while column (5) shows the measurements for this star available in Høg et al. (2000) and the NASA/IPAC Infrared Science Archive (2008) archive. Columns (6) and (7) show the synthetic colors measured for the Rieke et al. (2008) and Engelke et al. (2010) templates, respectively. The average differences between the synthetic and observed colors of Sirius and BD+60 1753 are $-0.006 \pm 0.010$ and and $0.007 \pm 0.028$, respectively. The mean difference between the synthetic colors measured for the four templates and the Vega SED are $\lesssim 0.018 \mathrm{mag}$, with a dispersion of the same order of magnitude ( $\lesssim 0.024 \mathrm{mag})$. These results suggest that the calibration uncertainty introduced by using the CALSPEC spectrum of Vega is $\sim 2 \%$.

In this work the vegamag magnitudes are calculated using the Vega SED of Bohlin (2014) (alpha_lyr_stis_008.fits in the STScI CALSPEC database), assuming a Vega magnitude of $V=0.03$ (BM12).

\section{The Solar Spectrum}

The solar SED used here also combines observations with model spectra. The observed spectrum is a composite calculated by Haberreiter et al. (2017) using data from over 20 space-based instruments for an arbitrary date (2008 December 19, JDN $=2454820$ ) during the solar minimum. Spectra for other dates around the solar minimum show no significant change relative to the spectrum adopted here. Haberreiter et al. (2017) used a probabilistic approach to combine observations at each timestep, weighting the spectra by their uncertainties and accounting for fluctuations over time between different instruments at the same wavelength. The absolute calibration is set using the ATLAS 3 composite spectrum of Thuillier et al. (2004), and constraining the Total Solar Irradiance (TSI) to the value measured for each day by Dudok de Wit et al. (2017). The observed composite ends at $\sim 2.0 \mu \mathrm{m}$, and to extend the SED into the infrared; the model spectra of Fontenla et al. (2011) 
Rieke et al. 2008

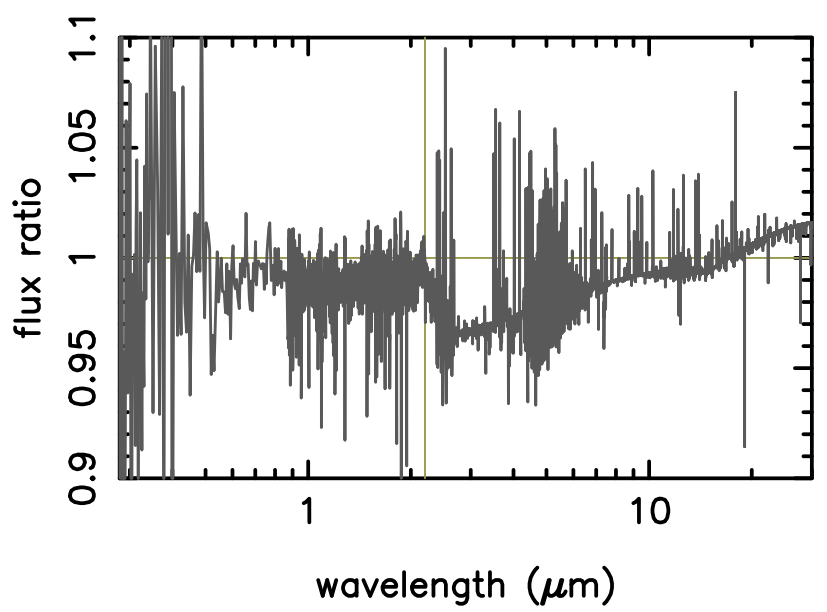

Woods et al. 2009

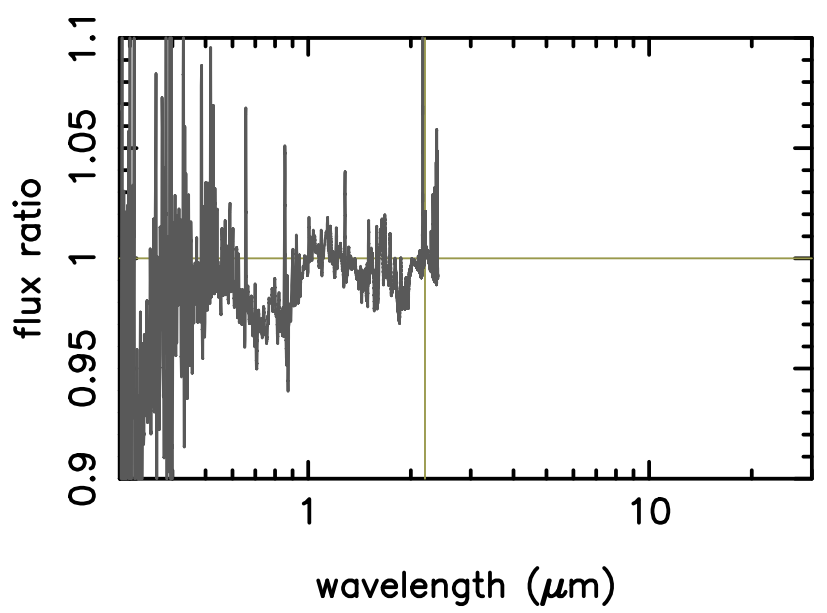

Atlas3

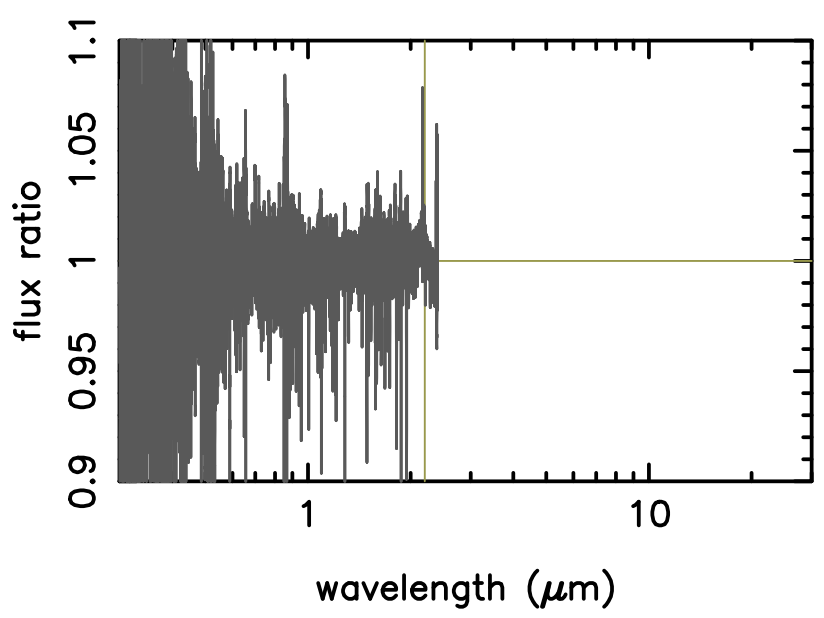

P330E

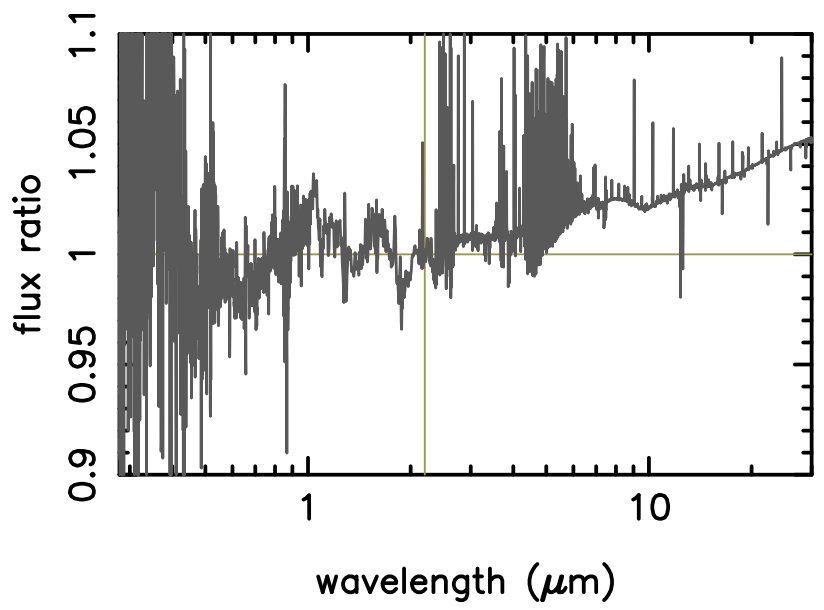

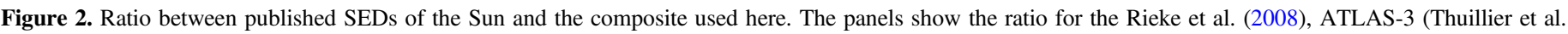

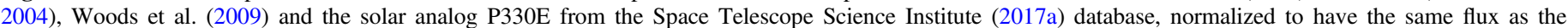

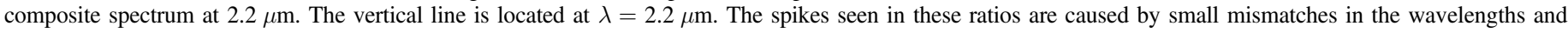

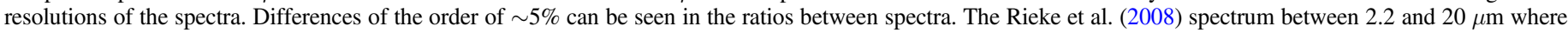

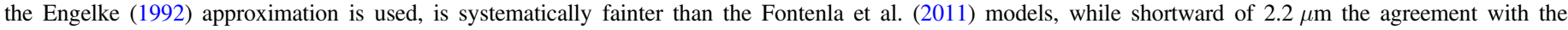
composite adopted here is very good. The spectrum of $\mathrm{P} 330 \mathrm{E}$ is systematically brighter than the composite used here for wavelengths longer than $\sim 2 \mu \mathrm{m}$.

and Kurucz (2011) are used. The Fontenla et al. (2011) model uses the Solar Irradiance Physical Modeling system to produce solar irradiance spectra from 0.012 to $100 \mu \mathrm{m}$ through a combination of non-LTE models with semiempirical physical models derived from observed spectra to produce the solar SED. The Fontenla et al. (2011) spectrum was scaled by the bi-weight average (Beers et al. 1990) ratio between the Haberreiter et al. (2017) composite and the model for wavelengths between $1.8 \mu \mathrm{m}$ and $2.0 \mu \mathrm{m}$ $(1.0299 \pm 0.0074)$. Beyond $100 \mu \mathrm{m}$, the special Kurucz model at $R=5000$ calculated for the CALSPEC database (Sun_mod_001.fits, Kurucz 2011) is used, and to eliminate any discontinuities in the transition between source spectra, the bi-weight ratio between the re-normalized Fontenla et al. (2011) and Kurucz models, calculated between 90 and $100.0 \mu \mathrm{m}(0.9940 \pm 0.0073)$, was used to scale the latter. Figure 1 shows the composite spectrum.
Figure 2 shows the ratios between this composite spectrum with other determinations in the literature-Rieke et al. (2008), Thuillier et al. (2004), Woods et al. (2009). Also shown is a comparison with the solar analog P330E after scaling its spectrum to have the same flux density as the composite at $2.2 \mu \mathrm{m}\left(8.58593 \mathrm{erg} \mathrm{cm}^{-2} \mathrm{~s}^{-1} \AA^{-1}\right)$. The ratios being plotted are calculated at each tabulated wavelength of the source spectrum and estimating the composite's flux using linear interpolation. The SED ratios are very close to 1 $(0.998,1.005,0.993$, and 1.017 for Rieke et al. (2008), Thuillier et al. (2004), Woods et al. (2009) and P330E, respectively) and dispersions of 0.037 or better, in all cases using the bi-weight estimator. The rms fluctuations range from 0.02 for the Rieke et al. (2008) solar spectrum, to 0.22 in the case of P330E.

A comparison between colors estimated using the solar composite spectrum with measurements by Ramírez et al. (2012) 
Table 2

Colors of the Solar Composite Compared to Solar Analogs

\begin{tabular}{|c|c|c|c|c|c|}
\hline Color & Composite & Solar Analogs & Difference & Rieke et al. (2008) SED & Difference \\
\hline$U-B$ & 0.164 & $0.166^{\mathrm{a}}$ & -0.002 & 0.138 & 0.026 \\
\hline$B-V$ & 0.629 & $0.653^{\mathrm{a}}$ & -0.024 & 0.629 & 0.000 \\
\hline$V-R$ & 0.387 & $0.352^{\mathrm{a}}$ & 0.035 & 0.388 & -0.001 \\
\hline$V-I$ & 0.712 & $0.702^{\mathrm{a}}$ & 0.010 & 0.717 & -0.005 \\
\hline$V-2 M A S S \_J$ & 1.145 & $1.198^{\mathrm{b}}$ & -0.053 & 1.143 & 0.002 \\
\hline$V$-2MASS_H & 1.494 & $1.484^{\mathrm{b}}$ & 0.010 & 1.492 & 0.002 \\
\hline$V-2 M A S S \_K s$ & 1.542 & $1.560^{\mathrm{b}}$ & -0.018 & 1.545 & -0.003 \\
\hline$V$-WISE_1 & 1.553 & $1.608^{b}$ & -0.055 & 1.530 & 0.023 \\
\hline$V$-WISE_2 & 1.530 & $1.563^{\mathrm{b}}$ & -0.033 & 1.515 & 0.015 \\
\hline$V$-WISE_3 & 1.549 & $1.552^{\mathrm{b}}$ & -0.003 & 1.551 & -0.002 \\
\hline$V$-WISE_4 & 1.539 & $1.604^{\mathrm{b}}$ & -0.065 & 1.559 & -0.020 \\
\hline
\end{tabular}

Notes.

${ }^{a}$ Ramírez et al. (2012).

${ }^{\text {b }}$ Casagrande et al. (2012).

and Casagrande et al. (2012) of solar analogs is presented in Table 2. The average difference in colors for (composite-solar analogs) is $-0.018 \pm 0.030 \mathrm{mag}$, suggesting that the composite spectrum shows consistent measurements both in the UV-visible and the infrared. Table 2 also compares the colors of the new composite with measurements using the Rieke et al. (2008) solar model, which combines the Thuillier et al. (2003) SED with the Engelke (1992) approximation for the near to mid-infrared. The (composite-Rieke et al. 2008) differences are $-0.003 \pm 0.013 \mathrm{mag}$, though these measurements may not be completely independent as Haberreiter et al. (2017) use the Thuillier et al. (2004) spectrum to set the absolute calibration of the solar spectrum.

The estimated errors in the solar magnitudes change as a function of wavelength due to the uncertainty on the absolute calibration using the Vega and Solar SED 2\%-3\% (Rieke et al. 2008; Bohlin 2014). When these are added in quadrature it results in uncertainties $\sim 3 \%$ over the range covered by solar analogs. These can become larger $(\sim 5 \%)$ as one transitions toward the mid-infrared due to the difficulty in calibrating the space-based instruments in this wavelength range, e.g., Fontenla et al. (2011).

To derive the Sun's absolute magnitudes, the IAU 2012 definitions of the astronomical unit (au) (Prša et al. 2016) and parsec were used, giving a distance modulus for the Sun of $-31.5721 \mathrm{mag}$. To rationalize the use of solar constants, the IAU in 2015 adopted a nominal value for the Sun's luminosity $L_{\odot}=3.828 \times 10^{8} \mathrm{~W}$ (Prša et al. 2016), which corresponds to an average TSI of $1361 \mathrm{~W} \mathrm{~m}^{-2}$ at 1 au and an absolute bolometric magnitude of $M_{\mathrm{Bol}}=4.74$.

The columns of Table 3 contain the following: (1) the filter; (2)-(4) the absolute magnitude of the Sun in the vegamag, AB, and ST systems, respectively; (5)-(7) the apparent magnitude in vegamag, $\mathrm{AB}$, and $\mathrm{ST}$; (8) and (9) tabulated offsets between the vegamag and $\mathrm{AB}$ and vegamag and ST systems; (10) the pivot wavelength; and (11) the source of the throughput curves, identified in the table notes.

\section{Summary}

This work uses the dust-free composite spectrum of Vega with the absolute calibration set by Sirius, both from Bohlin
(2014), to calculate a table with the absolute magnitude of the Sun and the conversion between the vegamag and the $\mathrm{AB}$ and ST systems for several filters used in ground-based and space-based observatories. The solar SED used in this paper is a composite combining space-based spectra of the Sun from the ultraviolet to the near-infrared (Haberreiter et al. 2017), with models of the solar atmosphere out to $300 \mu \mathrm{m}$ (Fontenla et al. 2011; Kurucz 2011). For the set of Johnson $(U, B, V)$ and Cousins ( $R$ and $I)$ filters, which were originally characterized using photoelectric photometry, filter curves reconstructed using Monte Carlo methods by Mann \& von Braun (2015) are used. To verify the consistency of the synthetic spectra measured using the composite spectra of Vega and the Sun, the colors measured for these SEDs are compared with photometric measurements of AV stellar templates and solar analogs, respectively. A comparison between colors calculated for the Vega SED and AV stars shows absolute offsets $<0.01 \mathrm{mag}$ and a dispersion $<0.03 \mathrm{mag}$, consistent with the estimated uncertainty at the 2\% level for the Vega SED by Bohlin (2014). The comparison between colors measured with the solar composite and the solar analogs of Ramírez et al. (2012) and Casagrande et al. (2012) shows an offset of $\sim-0.02 \pm$ $0.03 \mathrm{mag}$. Assuming the errors are equally distributed, this translates to an average uncertainty of $\sim 2 \%$ for the solar SED. Adding in quadrature the uncertainty in the calibration of both spectra translates to errors $\sim 3 \%-4 \%$ for the solar absolute magnitudes.

I thank George Rieke for suggestions and discussions. I also thank the anonymous referee and the editor Dr. Shadia Habbal, whose suggestions helped improve the presentation. Funding from the JWST/NIRCam contract NAS5-02015 to the University of Arizona, the use of the NASA/SAO ADS, the NASA/IPAC Infrared Science Archive, Simbad (at Strasbourg and Harvard), and the Mikulski Archive for Space Telescopes are gratefully acknowledged. This work uses data from the SOLID Project http://projects.pmodwrc.ch/solid/, which is funded by the European Community's Seventh Framework Programne (FP7 2012) under grant agreement no 313188.

Facilities: IRSA, MAST, JWST-Docs, GALEX, ADS, CDS. 
Table 3

Magnitudes of the Sun

\begin{tabular}{|c|c|c|c|c|c|c|c|c|c|c|}
\hline $\begin{array}{l}\text { Filter } \\
\text { (1) }\end{array}$ & $\begin{array}{c}\text { Abs } \\
\text { (Vega) } \\
\text { (2) }\end{array}$ & $\begin{array}{l}\text { Abs } \\
\text { (AB) } \\
(3)\end{array}$ & $\begin{array}{l}\text { Abs } \\
\text { (ST) } \\
(4)\end{array}$ & $\begin{array}{c}\text { App } \\
\text { (Vega) } \\
\text { (5) }\end{array}$ & $\begin{array}{l}\text { App } \\
\text { (AB) } \\
(6)\end{array}$ & $\begin{array}{l}\text { App } \\
\text { (ST) } \\
(7)\end{array}$ & $\begin{array}{c}\text { Vega } \\
(\mathrm{AB}) \\
(8)\end{array}$ & $\begin{array}{l}\text { Vega } \\
\text { (ST) } \\
(9)\end{array}$ & $\begin{array}{c}\lambda_{\text {pivot }} \\
(\mu \mathrm{m}) \\
(10)\end{array}$ & $\begin{array}{c}\text { Source } \\
\text { (11) }\end{array}$ \\
\hline Johnson_U & 5.61 & 6.33 & 5.42 & -25.97 & -25.25 & -26.15 & 0.721 & -0.183 & 0.3611 & 1 \\
\hline Johnson_B & 5.44 & 5.31 & 4.84 & -26.13 & -26.26 & -26.74 & -0.128 & -0.605 & 0.4396 & 1 \\
\hline Johnson_V & 4.81 & 4.80 & 4.81 & -26.76 & -26.77 & -26.76 & -0.013 & 0.001 & 0.5511 & 1 \\
\hline Cousins_R & 4.43 & 4.60 & 5.00 & -27.15 & -26.97 & -26.57 & 0.178 & 0.578 & 0.6582 & 1 \\
\hline Cousins_I & 4.10 & 4.51 & 5.35 & -27.47 & -27.06 & -26.22 & 0.414 & 1.247 & 0.8034 & 1 \\
\hline Tycho_Bt & 5.58 & 5.48 & 4.91 & -25.99 & -26.09 & -26.66 & -0.097 & -0.667 & 0.4212 & 1 \\
\hline Tycho_Vt & 4.88 & 4.85 & 4.79 & -26.69 & -26.72 & -26.78 & -0.035 & -0.091 & 0.5335 & 1 \\
\hline Hipparcos_Hp & 4.87 & 4.87 & 4.88 & -26.70 & -26.70 & -26.69 & -0.002 & 0.011 & 0.5508 & 1 \\
\hline 2MASS_J & 3.67 & 4.54 & 6.31 & -27.90 & -27.03 & -25.26 & 0.870 & 2.644 & 1.2393 & 2 \\
\hline 2MASS_H & 3.32 & 4.66 & 7.06 & -28.25 & -26.91 & -24.51 & 1.344 & 3.739 & 1.6495 & 2 \\
\hline 2MASS_Ks & 3.27 & 5.08 & 8.07 & -28.30 & -26.49 & -23.50 & 1.814 & 4.798 & 2.1638 & 2 \\
\hline SDSS_u & 5.49 & 6.39 & 5.45 & -26.08 & -25.18 & -26.12 & 0.900 & -0.037 & 0.3556 & 3 \\
\hline SDSS_g & 5.23 & 5.11 & 4.78 & -26.34 & -26.47 & -26.80 & -0.125 & -0.456 & 0.4702 & 3 \\
\hline SDSS_r & 4.53 & 4.65 & 4.91 & -27.04 & -26.93 & -26.66 & 0.119 & 0.380 & 0.6176 & 3 \\
\hline SDSS_i & 4.19 & 4.53 & 5.21 & -27.38 & -27.05 & -26.37 & 0.332 & 1.012 & 0.7490 & 3 \\
\hline SDSS_z & 4.01 & 4.50 & 5.57 & -27.56 & -27.07 & -26.00 & 0.494 & 1.560 & 0.8947 & 3 \\
\hline DES_u & 5.83 & 6.14 & 5.38 & -25.74 & -25.44 & -26.20 & 0.307 & -0.452 & 0.3859 & 4 \\
\hline DES_g & 5.17 & 5.05 & 4.78 & -26.41 & -26.52 & -26.80 & -0.114 & -0.391 & 0.4820 & 4 \\
\hline DES_r & 4.45 & 4.61 & 4.96 & -27.12 & -26.96 & -26.61 & 0.159 & 0.505 & 0.6423 & 4 \\
\hline DES_i & 4.14 & 4.52 & 5.29 & -27.43 & -27.05 & -26.28 & 0.382 & 1.152 & 0.7807 & 4 \\
\hline DES_z & 4.01 & 4.50 & 5.62 & -27.56 & -27.07 & -25.95 & 0.493 & 1.610 & 0.9158 & 4 \\
\hline DES_Y & 3.96 & 4.50 & 5.78 & -27.61 & -27.07 & -25.79 & 0.540 & 1.819 & 0.9866 & 4 \\
\hline PS1_g & 5.14 & 5.03 & 4.77 & -26.43 & -26.54 & -26.80 & -0.112 & -0.376 & 0.4849 & 5 \\
\hline $\mathrm{PS} 1 \_\mathrm{r}$ & 4.53 & 4.64 & 4.92 & -27.05 & -26.93 & -26.66 & 0.120 & 0.390 & 0.6201 & 5 \\
\hline PS1_i & 4.18 & 4.52 & 5.22 & -27.39 & -27.05 & -26.35 & 0.339 & 1.033 & 0.7535 & 5 \\
\hline PS1_z & 4.02 & 4.51 & 5.50 & -27.55 & -27.07 & -26.07 & 0.483 & 1.482 & 0.8674 & 5 \\
\hline PS1_Y & 3.99 & 4.50 & 5.73 & -27.59 & -27.07 & -25.85 & 0.515 & 1.741 & 0.9628 & 5 \\
\hline cfhtls_u & 5.70 & 6.04 & 5.25 & -25.87 & -25.53 & -26.33 & 0.336 & -0.455 & 0.3803 & 6 \\
\hline cfhtls_g & 5.15 & 5.03 & 4.77 & -26.42 & -26.54 & -26.80 & -0.116 & -0.382 & 0.4844 & 6 \\
\hline cfhtls_r & 4.50 & 4.64 & 4.92 & -27.07 & -26.94 & -26.65 & 0.131 & 0.417 & 0.6248 & 6 \\
\hline cfhtls_i & 4.16 & 4.52 & 5.26 & -27.41 & -27.05 & -26.32 & 0.362 & 1.096 & 0.7678 & 6 \\
\hline cfhtls_z & 4.02 & 4.51 & 5.55 & -27.56 & -27.07 & -26.02 & 0.490 & 1.535 & 0.8859 & 6 \\
\hline CFHT_12kx8k_B & 5.43 & 5.28 & 4.80 & -26.14 & -26.30 & -26.77 & -0.157 & -0.632 & 0.4399 & 7 \\
\hline CFHT_12kx8k_R & 4.39 & 4.59 & 5.00 & -27.18 & -26.98 & -26.57 & 0.196 & 0.605 & 0.6610 & 7 \\
\hline CFHT_12kx8k_I & 4.10 & 4.51 & 5.38 & -27.47 & -27.06 & -26.19 & 0.415 & 1.282 & 0.8159 & 7 \\
\hline UKIRT_z & 4.02 & 4.51 & 5.54 & -27.56 & -27.07 & -26.03 & 0.489 & 1.526 & 0.8826 & 8 \\
\hline UKIRT_Y & 3.92 & 4.51 & 5.88 & -27.66 & -27.07 & -25.69 & 0.591 & 1.966 & 1.0315 & 8 \\
\hline UKIRT_J & 3.65 & 4.54 & 6.33 & -27.92 & -27.03 & -25.24 & 0.891 & 2.684 & 1.2502 & 8 \\
\hline UKIRT_H & 3.33 & 4.66 & 7.03 & -28.25 & -26.92 & -24.54 & 1.329 & 3.705 & 1.6360 & 8 \\
\hline UKIRT_K & 3.27 & 5.12 & 8.14 & -28.30 & -26.45 & -23.43 & 1.848 & 4.874 & 2.2060 & 8 \\
\hline LSST_u & 5.65 & 6.27 & 5.40 & -25.93 & -25.30 & -26.17 & 0.627 & -0.244 & 0.3665 & 9 \\
\hline LSST_g & 5.17 & 5.06 & 4.77 & -26.40 & -26.52 & -26.80 & -0.116 & -0.399 & 0.4808 & 9 \\
\hline LSST_r & 4.52 & 4.64 & 4.92 & -27.05 & -26.93 & -26.66 & 0.121 & 0.395 & 0.6210 & 9 \\
\hline LSST_i & 4.18 & 4.52 & 5.22 & -27.39 & -27.05 & -26.35 & 0.340 & 1.034 & 0.7537 & 9 \\
\hline LSST_z & 4.02 & 4.51 & 5.51 & -27.55 & -27.07 & -26.06 & 0.484 & 1.486 & 0.8686 & 9 \\
\hline LSST_y & 3.98 & 4.50 & 5.74 & -27.59 & -27.07 & -25.83 & 0.520 & 1.763 & 0.9705 & 9 \\
\hline Bessell_Murphy_U & 5.57 & 6.34 & 5.43 & -26.00 & -25.23 & -26.14 & 0.768 & -0.144 & 0.3597 & 10 \\
\hline Bessell_Murphy_B & 5.46 & 5.33 & 4.84 & -26.11 & -26.24 & -26.73 & -0.134 & -0.620 & 0.4378 & 10 \\
\hline Bessell_Murphy_V & 4.82 & 4.81 & 4.81 & -26.75 & -26.77 & -26.76 & -0.017 & -0.012 & 0.5489 & 10 \\
\hline Bessell_Murphy_R & 4.44 & 4.61 & 4.99 & -27.13 & -26.96 & -26.58 & 0.168 & 0.548 & 0.6524 & 10 \\
\hline Bessell_Murphy_I & 4.11 & 4.52 & 5.33 & -27.46 & -27.06 & -26.24 & 0.408 & 1.227 & 0.7984 & 10 \\
\hline Bessell_Murphy_Bt & 5.60 & 5.51 & 4.93 & -25.98 & -26.06 & -26.65 & -0.088 & -0.669 & 0.4190 & 10 \\
\hline Bessell_Murphy_Vt & 4.89 & 4.86 & 4.79 & -26.68 & -26.71 & -26.79 & -0.038 & -0.108 & 0.5300 & 10 \\
\hline Bessell_Murphy_Hp & 4.93 & 4.92 & 4.86 & -26.64 & -26.66 & -26.71 & -0.018 & -0.068 & 0.5349 & 10 \\
\hline Bessell_88_J & 3.67 & 4.54 & 6.30 & -27.90 & -27.03 & -25.27 & 0.866 & 2.632 & 1.2347 & 11 \\
\hline Bessell_88_H & 3.32 & 4.66 & 7.05 & -28.25 & -26.91 & -24.52 & 1.337 & 3.726 & 1.6450 & 11 \\
\hline Bessell_88_K & 3.27 & 5.09 & 8.07 & -28.30 & -26.49 & -23.50 & 1.815 & 4.802 & 2.1663 & 11 \\
\hline Bessell_88_L & 3.26 & 5.98 & 10.00 & -28.31 & -25.59 & -21.58 & 2.721 & 6.737 & 3.4797 & 11 \\
\hline Bessell_88_Lprime & 3.26 & 6.17 & 10.39 & -28.31 & -25.40 & -21.18 & 2.914 & 7.135 & 3.8247 & 11 \\
\hline Bessell_88_M & 3.29 & 6.64 & 11.33 & -28.28 & -24.93 & -20.25 & 3.349 & 8.034 & 4.7347 & 11 \\
\hline GALEX_FUV & 15.22 & 17.30 & 14.54 & -16.36 & -14.27 & -17.03 & 2.085 & -0.676 & 0.1535 & 12 \\
\hline GALEX_NUV & 8.53 & 10.16 & 8.28 & -23.04 & -21.41 & -23.30 & 1.629 & -0.253 & 0.2301 & 12 \\
\hline
\end{tabular}


Table 3

(Continued)

\begin{tabular}{|c|c|c|c|c|c|c|c|c|c|c|}
\hline $\begin{array}{l}\text { Filter } \\
\text { (1) }\end{array}$ & $\begin{array}{c}\text { Abs } \\
\text { (Vega) } \\
(2)\end{array}$ & $\begin{array}{c}\text { Abs } \\
(\mathrm{AB}) \\
(3)\end{array}$ & $\begin{array}{l}\text { Abs } \\
\text { (ST) } \\
\text { (4) }\end{array}$ & $\begin{array}{c}\text { App } \\
\text { (Vega) } \\
(5)\end{array}$ & $\begin{array}{c}\text { App } \\
\text { (AB) } \\
(6)\end{array}$ & $\begin{array}{l}\text { App } \\
\text { (ST) } \\
(7)\end{array}$ & $\begin{array}{c}\text { Vega } \\
(\mathrm{AB}) \\
(8)\end{array}$ & $\begin{array}{l}\text { Vega } \\
\text { (ST) } \\
(9)\end{array}$ & $\begin{array}{c}\lambda_{\text {pivot }} \\
(\mu \mathrm{m}) \\
(10)\end{array}$ & $\begin{array}{r}\text { Source } \\
\text { (11) }\end{array}$ \\
\hline$\overline{\text { WISE_1 }}$ & 3.26 & 5.91 & 9.87 & -28.31 & -25.66 & -21.70 & 2.655 & 6.614 & 3.3897 & 13 \\
\hline WISE_2 & 3.28 & 6.57 & 11.22 & -28.29 & -25.00 & -20.36 & 3.291 & 7.932 & 4.6406 & 13 \\
\hline WISE_3 & 3.26 & 8.48 & 15.28 & -28.31 & -23.09 & -16.29 & 5.215 & 12.019 & 12.5705 & 13 \\
\hline WISE_4 & 3.27 & 9.88 & 17.93 & -28.30 & -21.70 & -13.65 & 6.602 & 14.652 & 22.3142 & 13 \\
\hline IRAS12 & 3.26 & 8.30 & 14.89 & -28.31 & -23.27 & -16.69 & 5.037 & 11.621 & 11.3562 & 13 \\
\hline IRAS25 & 3.27 & 9.92 & 18.09 & -28.30 & -21.65 & -13.48 & 6.646 & 14.819 & 23.6079 & 13 \\
\hline IRAS60 & 3.28 & 11.90 & 22.12 & -28.29 & -19.67 & -9.46 & 8.621 & 18.833 & 60.3699 & 13 \\
\hline IRAS100 & 3.29 & 13.14 & 24.47 & -28.28 & -18.43 & -7.10 & 9.854 & 21.186 & 101.1267 & 13 \\
\hline IRAC_3.6 & 3.26 & 6.02 & 10.08 & -28.31 & -25.56 & -21.50 & 2.758 & 6.817 & 3.5508 & 13 \\
\hline IRAC_4.5 & 3.28 & 6.51 & 11.08 & -28.29 & -25.06 & -20.49 & 3.232 & 7.804 & 4.4960 & 13 \\
\hline IRAC_5.8 & 3.28 & 7.00 & 12.09 & -28.30 & -24.58 & -19.48 & 3.720 & 8.816 & 5.7245 & 13 \\
\hline IRAC_ 8.0 & 3.26 & 7.62 & 13.41 & -28.31 & -23.95 & -18.16 & 4.360 & 10.152 & 7.8842 & 13 \\
\hline IRS_16 & 3.27 & 9.11 & 16.42 & -28.31 & -22.47 & -15.15 & 5.839 & 13.157 & 15.9222 & 13 \\
\hline IRS_22 & 3.27 & 9.86 & 17.92 & -28.30 & -21.72 & -13.65 & 6.584 & 14.650 & 22.4704 & 14 \\
\hline MIPS_24 & 3.27 & 10.01 & 18.19 & -28.30 & -21.57 & -13.38 & 6.731 & 14.918 & 23.7592 & 14 \\
\hline MIPS_70 & 3.29 & 12.40 & 23.00 & -28.28 & -19.17 & -8.58 & 9.114 & 19.708 & 71.9861 & 14 \\
\hline MIPS_160 & 3.29 & 14.15 & 26.42 & -28.28 & -17.43 & -5.15 & 10.857 & 23.137 & 156.4274 & 14 \\
\hline ACS_F330W & 5.34 & 6.43 & 5.47 & -26.24 & -25.14 & -26.10 & 1.097 & 0.139 & 0.3521 & 14 \\
\hline ACS_F410W & 5.70 & 5.67 & 5.02 & -25.87 & -25.90 & -26.55 & -0.033 & -0.680 & 0.4064 & 14 \\
\hline ACS_F435W & 5.48 & 5.35 & 4.84 & -26.09 & -26.22 & -26.73 & -0.129 & -0.639 & 0.4328 & 14 \\
\hline ACS_F475W & 5.21 & 5.09 & 4.78 & -26.36 & -26.49 & -26.80 & -0.122 & -0.432 & 0.4747 & 14 \\
\hline ACS_F555W & 4.87 & 4.84 & 4.79 & -26.71 & -26.74 & -26.78 & -0.030 & -0.076 & 0.5361 & 14 \\
\hline ACS_F606W & 4.66 & 4.72 & 4.89 & -26.92 & -26.85 & -26.68 & 0.063 & 0.233 & 0.5922 & 14 \\
\hline ACS_F625W & 4.49 & 4.63 & 4.94 & -27.08 & -26.94 & -26.64 & 0.140 & 0.448 & 0.6312 & 14 \\
\hline ACS_F775W & 4.16 & 4.52 & 5.26 & -27.42 & -27.05 & -26.31 & 0.364 & 1.103 & 0.7694 & 14 \\
\hline ACS_F814W & 4.12 & 4.52 & 5.36 & -27.46 & -27.06 & -26.22 & 0.400 & 1.239 & 0.8059 & 14 \\
\hline ACS_F850LP & 4.01 & 4.50 & 5.59 & -27.56 & -27.07 & -25.98 & 0.494 & 1.577 & 0.9016 & 14 \\
\hline WFC3_F218W & 9.09 & 10.74 & 8.79 & -22.48 & -20.83 & -22.78 & 1.654 & -0.298 & 0.2229 & 14 \\
\hline WFC3_F225W & 8.51 & 10.13 & 8.32 & -23.06 & -21.44 & -23.25 & 1.625 & -0.191 & 0.2372 & 14 \\
\hline WFC3_F336W & 5.49 & 6.64 & 5.58 & -26.09 & -24.93 & -25.99 & 1.158 & 0.094 & 0.3355 & 14 \\
\hline WFC3_F390W & 5.66 & 5.85 & 5.12 & -25.91 & -25.73 & -26.45 & 0.187 & -0.536 & 0.3924 & 14 \\
\hline WFC3_F438W & 5.50 & 5.32 & 4.81 & -26.07 & -26.25 & -26.76 & -0.178 & -0.690 & 0.4326 & 14 \\
\hline WFC3_F475W & 5.19 & 5.07 & 4.77 & -26.38 & -26.50 & -26.80 & -0.122 & -0.419 & 0.4774 & 14 \\
\hline WFC3_F555W & 4.91 & 4.86 & 4.79 & -26.67 & -26.72 & -26.78 & -0.048 & -0.116 & 0.5308 & 14 \\
\hline WFC3_F606W & 4.67 & 4.73 & 4.88 & -26.91 & -26.85 & -26.69 & 0.059 & 0.217 & 0.5887 & 14 \\
\hline WFC3_F625W & 4.52 & 4.64 & 4.92 & -27.06 & -26.93 & -26.65 & 0.124 & 0.409 & 0.6241 & 14 \\
\hline WFC3_F775W & 4.16 & 4.52 & 5.25 & -27.41 & -27.05 & -26.33 & 0.357 & 1.083 & 0.7648 & 14 \\
\hline WFC3_F814W & 4.12 & 4.52 & 5.35 & -27.45 & -27.06 & -26.22 & 0.395 & 1.226 & 0.8030 & 14 \\
\hline WFC3_F098m & 3.96 & 4.50 & 5.78 & -27.61 & -27.07 & -25.79 & 0.538 & 1.816 & 0.9864 & 14 \\
\hline WFC3_F105W & 3.89 & 4.51 & 5.94 & -27.68 & -27.06 & -25.63 & 0.622 & 2.046 & 1.0551 & 14 \\
\hline WFC3_F125W & 3.66 & 4.54 & 6.33 & -27.91 & -27.03 & -25.24 & 0.877 & 2.667 & 1.2486 & 14 \\
\hline WFC3_F140W & 3.51 & 4.56 & 6.59 & -28.06 & -27.01 & -24.98 & 1.052 & 3.079 & 1.3922 & 14 \\
\hline WFC3_F160W & 3.37 & 4.60 & 6.84 & -28.20 & -26.97 & -24.73 & 1.228 & 3.469 & 1.5370 & 14 \\
\hline WFPC2_F218W & 9.17 & 10.83 & 8.86 & -22.40 & -20.74 & -22.72 & 1.657 & -0.316 & 0.2207 & 15 \\
\hline WFPC2_F300W & 6.10 & 7.40 & 6.09 & -25.48 & -24.17 & -25.48 & 1.307 & -0.005 & 0.2992 & 15 \\
\hline WFPC2_F450W & 5.31 & 5.20 & 4.80 & -26.26 & -26.37 & -26.77 & -0.110 & -0.509 & 0.4556 & 15 \\
\hline WFPC2_F555W & 4.84 & 4.82 & 4.81 & -26.73 & -26.75 & -26.77 & -0.025 & -0.038 & 0.5442 & 15 \\
\hline WFPC2_F606W & 4.62 & 4.70 & 4.90 & -26.95 & -26.87 & -26.67 & 0.077 & 0.276 & 0.6001 & 15 \\
\hline WFPC2_F702W & 4.33 & 4.57 & 5.08 & -27.24 & -27.00 & -26.49 & 0.240 & 0.748 & 0.6919 & 15 \\
\hline WFPC2_F814W & 4.12 & 4.52 & 5.34 & -27.45 & -27.05 & -26.23 & 0.392 & 1.216 & 0.8002 & 15 \\
\hline NIC2_F110W & 3.82 & 4.52 & 6.08 & -27.75 & -27.05 & -25.49 & 0.704 & 2.265 & 1.1235 & 15 \\
\hline NIC2_F160W & 3.35 & 4.64 & 6.97 & -28.22 & -26.93 & -24.60 & 1.286 & 3.618 & 1.6030 & 15 \\
\hline NIC3_F110W & 3.82 & 4.52 & 6.08 & -27.75 & -27.05 & -25.50 & 0.701 & 2.255 & 1.1200 & 15 \\
\hline NIC3_F160W & 3.35 & 4.64 & 6.97 & -28.22 & -26.93 & -24.60 & 1.287 & 3.621 & 1.6042 & 15 \\
\hline NIRCAM_F070W & 4.29 & 4.56 & 5.10 & -27.28 & -27.02 & -26.47 & 0.264 & 0.811 & 0.7046 & 15 \\
\hline NIRCAM_F090W & 4.02 & 4.50 & 5.59 & -27.56 & -27.07 & -25.98 & 0.488 & 1.573 & 0.9025 & 15 \\
\hline NIRCAM_F115W & 3.77 & 4.53 & 6.15 & -27.80 & -27.05 & -25.43 & 0.753 & 2.373 & 1.1543 & 15 \\
\hline NIRCAM_F140M & 3.48 & 4.56 & 6.60 & -28.09 & -27.02 & -24.97 & 1.079 & 3.126 & 1.4053 & 15 \\
\hline NIRCAM_F150W & 3.41 & 4.59 & 6.78 & -28.16 & -26.98 & -24.79 & 1.182 & 3.371 & 1.5007 & 15 \\
\hline NIRCAM_F150W2 & 3.50 & 4.70 & 7.11 & -28.07 & -26.87 & -24.46 & 1.203 & 3.610 & 1.6588 & 15 \\
\hline NIRCAM_F162M & 3.32 & 4.65 & 7.01 & -28.25 & -26.93 & -24.56 & 1.328 & 3.693 & 1.6272 & 15 \\
\hline NIRCAM_F164N & 3.29 & 4.66 & 7.05 & -28.28 & -26.91 & -24.53 & 1.368 & 3.756 & 1.6445 & 15 \\
\hline
\end{tabular}


Table 3

(Continued)

\begin{tabular}{|c|c|c|c|c|c|c|c|c|c|c|}
\hline $\begin{array}{l}\text { Filter } \\
\text { (1) }\end{array}$ & $\begin{array}{c}\text { Abs } \\
\text { (Vega) } \\
(2)\end{array}$ & $\begin{array}{c}\text { Abs } \\
(\mathrm{AB}) \\
(3)\end{array}$ & $\begin{array}{c}\text { Abs } \\
(\mathrm{ST}) \\
(4)\end{array}$ & $\begin{array}{c}\text { App } \\
\text { (Vega) } \\
(5)\end{array}$ & $\begin{array}{c}\text { App } \\
(\mathrm{AB}) \\
(6)\end{array}$ & $\begin{array}{l}\text { App } \\
\text { (ST) } \\
(7)\end{array}$ & $\begin{array}{c}\text { Vega } \\
(\mathrm{AB}) \\
(8)\end{array}$ & $\begin{array}{l}\text { Vega } \\
\text { (ST) } \\
(9)\end{array}$ & $\begin{array}{c}\lambda_{\text {pivot }} \\
(\mu \mathrm{m}) \\
(10)\end{array}$ & $\begin{array}{c}\text { Source } \\
\text { (11) }\end{array}$ \\
\hline NIRCAM_F182M & 3.28 & 4.81 & 7.45 & -28.29 & -26.76 & -24.12 & 1.534 & 4.172 & 1.8452 & 15 \\
\hline NIRCAM_F187N & 3.25 & 4.85 & 7.52 & -28.33 & -26.72 & -24.05 & 1.600 & 4.272 & 1.8739 & 15 \\
\hline NIRCAM_F200W & 3.28 & 4.93 & 7.73 & -28.30 & -26.64 & -23.84 & 1.652 & 4.453 & 1.9886 & 15 \\
\hline NIRCAM_F200W & 3.28 & 4.93 & 7.73 & -28.30 & -26.64 & -23.84 & 1.652 & 4.453 & 1.9886 & 15 \\
\hline NIRCAM_F210M & 3.27 & 5.03 & 7.94 & -28.30 & -26.54 & -23.63 & 1.757 & 4.671 & 2.0955 & 15 \\
\hline NIRCAM_F250M & 3.27 & 5.37 & 8.67 & -28.30 & -26.21 & -22.91 & 2.093 & 5.393 & 2.5032 & 15 \\
\hline NIRCAM_F277W & 3.26 & 5.53 & 9.04 & -28.31 & -26.04 & -22.53 & 2.265 & 5.779 & 2.7618 & 15 \\
\hline NIRCAM_F300M & 3.26 & 5.69 & 9.37 & -28.31 & -25.88 & -22.20 & 2.429 & 6.115 & 2.9892 & 15 \\
\hline NIRCAM_F322W2 & 3.26 & 5.77 & 9.63 & -28.31 & -25.80 & -21.95 & 2.509 & 6.365 & 3.2320 & 15 \\
\hline NIRCAM_F323N & 3.26 & 5.84 & 9.70 & -28.31 & -25.73 & -21.87 & 2.583 & 6.441 & 3.2369 & 15 \\
\hline NIRCAM_F335M & 3.26 & 5.92 & 9.86 & -28.31 & -25.66 & -21.71 & 2.658 & 6.599 & 3.3621 & 15 \\
\hline NIRCAM_F356W & 3.26 & 6.02 & 10.09 & -28.31 & -25.55 & -21.48 & 2.763 & 6.833 & 3.5684 & 15 \\
\hline NIRCAM_F405N & 3.24 & 6.30 & 10.65 & -28.33 & -25.27 & -20.93 & 3.058 & 7.404 & 4.0517 & 15 \\
\hline NIRCAM_F410M & 3.26 & 6.31 & 10.67 & -28.32 & -25.27 & -20.90 & 3.049 & 7.411 & 4.0822 & 15 \\
\hline NIRCAM_F430M & 3.27 & 6.41 & 10.88 & -28.31 & -25.16 & -20.69 & 3.147 & 7.613 & 4.2813 & 15 \\
\hline NIRCAM_F444W & 3.27 & 6.46 & 10.99 & -28.30 & -25.11 & -20.59 & 3.185 & 7.712 & 4.4040 & 15 \\
\hline NIRCAM_F460M & 3.29 & 6.60 & 11.23 & -28.28 & -24.97 & -20.34 & 3.308 & 7.943 & 4.6285 & 15 \\
\hline NIRCAM_F466N & 3.26 & 6.62 & 11.26 & -28.31 & -24.96 & -20.31 & 3.352 & 8.000 & 4.6544 & 15 \\
\hline NIRCAM_F470N & 3.29 & 6.63 & 11.30 & -28.28 & -24.94 & -20.27 & 3.341 & 8.013 & 4.7078 & 15 \\
\hline NIRCAM_F480M & 3.29 & 6.67 & 11.39 & -28.28 & -24.90 & -20.18 & 3.383 & 8.104 & 4.8167 & 15 \\
\hline MIRI_F560W & 3.28 & 6.97 & 12.03 & -28.29 & -24.60 & -19.54 & 3.693 & 8.756 & 5.6362 & 16 \\
\hline MIRI_F770W & 3.26 & 7.58 & 13.30 & -28.31 & -24.00 & -18.27 & 4.314 & 10.039 & 7.6428 & 16 \\
\hline MIRI_F1000W & 3.26 & 8.15 & 14.45 & -28.31 & -23.42 & -17.13 & 4.883 & 11.181 & 9.9544 & 16 \\
\hline MIRI_F1130W & 3.26 & 8.43 & 15.00 & -28.31 & -23.14 & -16.57 & 5.166 & 11.741 & 11.3087 & 16 \\
\hline MIRI_F1500W & 3.27 & 9.03 & 16.23 & -28.31 & -22.54 & -15.35 & 5.763 & 12.961 & 15.0651 & 16 \\
\hline MIRI_F1800W & 3.27 & 9.42 & 17.00 & -28.30 & -22.15 & -14.57 & 6.149 & 13.732 & 17.9865 & 16 \\
\hline MIRI_F2100W & 3.27 & 9.72 & 17.62 & -28.30 & -21.85 & -13.95 & 6.453 & 14.351 & 20.7950 & 16 \\
\hline MIRI_F2550W & 3.28 & 10.16 & 18.49 & -28.30 & -21.41 & -13.08 & 6.887 & 15.216 & 25.3639 & 16 \\
\hline NIRISSS_F090W & 4.02 & 4.50 & 5.59 & -27.56 & -27.07 & -25.98 & 0.488 & 1.575 & 0.9031 & 17 \\
\hline NIRISS_F115W & 3.78 & 4.53 & 6.14 & -27.79 & -27.05 & -25.43 & 0.747 & 2.358 & 1.1499 & 17 \\
\hline NIRISS_F140M & 3.48 & 4.56 & 6.60 & -28.09 & -27.02 & -24.97 & 1.078 & 3.123 & 1.4044 & 17 \\
\hline NIRISS_F150W & 3.41 & 4.59 & 6.77 & -28.16 & -26.98 & -24.81 & 1.173 & 3.352 & 1.4936 & 17 \\
\hline NIRISS_F158M & 3.35 & 4.62 & 6.93 & -28.23 & -26.95 & -24.64 & 1.277 & 3.582 & 1.5825 & 17 \\
\hline NIRISS_F200W & 3.28 & 4.93 & 7.74 & -28.30 & -26.64 & -23.83 & 1.656 & 4.461 & 1.9930 & 17 \\
\hline NIRISS_F277W & 3.27 & 5.53 & 9.04 & -28.30 & -26.05 & -22.53 & 2.258 & 5.774 & 2.7641 & 17 \\
\hline NIRISS_F356W & 3.26 & 6.03 & 10.11 & -28.31 & -25.54 & -21.46 & 2.769 & 6.854 & 3.5926 & 17 \\
\hline NIRISS_F380M & 3.26 & 6.17 & 10.39 & -28.31 & -25.40 & -21.18 & 2.908 & 7.128 & 3.8229 & 17 \\
\hline NIRISS_F430M & 3.27 & 6.40 & 10.87 & -28.30 & -25.17 & -20.70 & 3.130 & 7.595 & 4.2792 & 17 \\
\hline NIRISS_F444W & 3.27 & 6.47 & 11.00 & -28.30 & -25.11 & -20.57 & 3.191 & 7.729 & 4.4270 & 17 \\
\hline NIRISS_F480M & 3.29 & 6.66 & 11.38 & -28.28 & -24.91 & -20.19 & 3.366 & 8.086 & 4.8113 & 17 \\
\hline OMEGACAM_u & 5.46 & 6.34 & 5.43 & -26.11 & -25.23 & -26.15 & 0.881 & -0.035 & 0.3590 & 18 \\
\hline OMEGACAM_g & 5.21 & 5.09 & 4.77 & -26.36 & -26.48 & -26.80 & -0.126 & -0.442 & 0.4735 & 18 \\
\hline OMEGACAM_r & 4.50 & 4.63 & 4.93 & -27.07 & -26.94 & -26.64 & 0.133 & 0.429 & 0.6276 & 18 \\
\hline OMEGACAM_i & 4.20 & 4.53 & 5.21 & -27.38 & -27.05 & -26.36 & 0.331 & 1.013 & 0.7495 & 18 \\
\hline OMEGACAM_z & 4.01 & 4.51 & 5.55 & -27.56 & -27.07 & -26.03 & 0.493 & 1.534 & 0.8842 & 18 \\
\hline VIRCAM_Z & 4.02 & 4.51 & 5.56 & -27.56 & -27.07 & -26.01 & 0.491 & 1.546 & 0.8899 & 19 \\
\hline VIRCAM_Y & 3.93 & 4.51 & 5.87 & -27.64 & -27.07 & -25.70 & 0.577 & 1.940 & 1.0253 & 19 \\
\hline VIRCAM_H & 3.65 & 4.54 & 6.34 & -27.93 & -27.03 & -25.23 & 0.892 & 2.691 & 1.2535 & 19 \\
\hline VIRCAM_J & 3.32 & 4.66 & 7.05 & -28.25 & -26.91 & -24.53 & 1.335 & 3.721 & 1.6430 & 19 \\
\hline VIRCAM_Ks & 3.27 & 5.07 & 8.04 & -28.30 & -26.50 & -23.53 & 1.797 & 4.767 & 2.1494 & 19 \\
\hline SkyMapper_u & 5.33 & 6.32 & 5.40 & -26.24 & -25.25 & -26.17 & 0.989 & 0.073 & 0.3590 & 20 \\
\hline SkyMapper_v & 5.81 & 6.09 & 5.31 & -25.77 & -25.49 & -26.26 & 0.280 & -0.493 & 0.3836 & 20 \\
\hline SkyMapper_g & 5.03 & 4.94 & 4.78 & -26.55 & -26.63 & -26.79 & -0.082 & -0.247 & 0.5075 & 20 \\
\hline SkyMapper_r & 4.56 & 4.66 & 4.91 & -27.02 & -26.91 & -26.66 & 0.104 & 0.352 & 0.6138 & 20 \\
\hline SkyMapper_i & 4.14 & 4.52 & 5.28 & -27.43 & -27.05 & -26.29 & 0.377 & 1.137 & 0.7768 & 20 \\
\hline SkyMapper_z & 4.00 & 4.50 & 5.62 & -27.57 & -27.07 & -25.95 & 0.502 & 1.615 & 0.9143 & 20 \\
\hline
\end{tabular}

References: (1) Mann \& von Braun (2015), (2) Cohen et al. (2003), (3) Gunn et al. (1998), (4) National Optical Astronomy Observatories (2015), (5) Tonry et al. (2012), (6) Gwyn (2012), (7) N. Kaiser (2002, private communication), (8) Hewett et al. (2006), (9) https://github.com/lsst/throughputs/tree/master/baseline, (10) Bessell \& Murphy (2012), (11) Bessell \& Brett (1988), (12) Goddard Space Flight Center (2012), (13) Jarrett et al. (2011), (14) Gillett et al. (1984), (15) NASA/IPAC Infrared Science Archive (2008), (15) Space Telescope Science Institute (2017a), (16) Space Telescope Science Institute (2017b), (17) Space Telescope Science Institute (2017b). (18) https://www.eso. $\mathrm{org} / \mathrm{sci} /$ facilities/paranal/instruments/omegacam/tools.html, (19) https://www.eso.org/sci/facilities/paranal/instruments/vircam/inst.html, (20) Bessell et al. (2011). 


\section{Appendix}

\section{Filter Parameters}

As shown by Rieke et al. (2008) and BM12, there are a number of definitions used to characterize filter properties and frequently the names associated with these definitions these are inconsistent in the literature (BM12). For convenience, the expressions used to calculate the filter parameters are presented here and the reader is referred to Appendix E of Rieke et al. (2008), the appendix of Bessell \& Murphy (2012), and the review in Bohlin et al. (2014) for more detailed discussions on the determination, history, and naming of these definitions.

The following characteristic wavelengths are only dependent on the filter shape. The mean photon wavelength (Bessell \& Murphy 2012), also called mean wavelength by Tokunaga $\&$ Vacca (2005) and mean or effective wavelength by Rieke et al. (2008) is defined as

$$
\lambda_{\text {mean }}=\frac{\int R(\lambda) \lambda d \lambda}{\int R(\lambda) d \lambda} .
$$

The mean flux of a source within the band is defined as

$$
\left\langle f_{\lambda}\right\rangle=\frac{\int f_{\lambda} R(\lambda) \lambda d \lambda}{\int R(\lambda) \lambda d \lambda} .
$$

The nominal wavelength of Rieke et al. (2008) is called mean energy wavelength by Bessell \& Murphy (2012):

$$
\lambda_{n 1}=\frac{\int R(\lambda) \lambda^{2} d \lambda}{\int R(\lambda) \lambda d \lambda},
$$

while Reach et al. (2005) define the nominal wavelength as

$$
\lambda_{n 2}=\frac{\int R(\lambda) d \lambda}{\int \frac{R(\lambda) d \lambda}{\lambda}}
$$

and in both cases minimize the color correction in a given band (Reach et al. 2005; Rieke et al. 2008).

The pivot wavelength

$$
\lambda_{\text {pivot }}=\sqrt{\frac{\int R(\lambda) \lambda d \lambda}{\int \frac{R(\lambda) d \lambda}{\lambda}}}
$$

is the wavelength where $\left\langle f_{\lambda}\right\rangle \frac{\lambda_{\text {pivot }}^{2}}{c}=\left\langle f_{\nu}\right\rangle$, and $\left\langle f_{\lambda}\right\rangle$ or $\left\langle f_{\nu}\right\rangle$ are the mean flux density within the band.

The following characteristic wavelengths also take into account the flux density of the source $\left(f_{\lambda}\right)$. As noted by BM12, there is a multiplicity of definitions for the effective wavelength, and they propose this as the standard:

$$
\lambda_{\mathrm{eff}}=\frac{\int f_{\lambda}(\lambda) R(\lambda) \lambda^{2} d \lambda}{\int R f_{\lambda}(\lambda)(\lambda) \lambda d \lambda} .
$$

The wavelength where the monochromatic flux of a source is equivalent to the average flux of the source within the band is defined as the isophotal wavelength (Cohen et al. 1992;
Tokunaga \& Vacca 2005; Rieke et al. 2008; BM12) for Spitzer:

$$
f\left(\lambda_{\text {iso }}\right)=\left\langle f_{\lambda}\right\rangle .
$$

Because this measurement can be affected by the instrumental resolution and the presence of stellar lines (Rieke et al. 2008), when calculating the isophotal wavelength one may need to smooth the spectrum prior to the calculation (Bessell \& Murphy 2012), use a continuum model, or interpolate over spectral lines (Cohen et al. 1992; Rieke et al. 2008).

The bandwidth is defined as the integral of the normalized transmission (Budding 1993), and the following definition is adopted by Rieke et al. (2008) and Mann \& von Braun (2015, where it is called effective width):

$$
\mathrm{BW}=\frac{\int R(\lambda) d \lambda}{\max [R(\lambda)]}
$$

The average system response is

$$
\operatorname{Resp}=\frac{\int R(\lambda) d \lambda}{\int d \lambda}
$$

For the NIRCam filters tabulated in Space Telescope Science Institute (2017b), an effective response is adopted where

$$
R_{\mathrm{eff}}=\frac{\int_{\lambda-\text { pivot }-\mathrm{BW} / 2}^{\lambda \_ \text {pivot }+\mathrm{BW} / 2} R(\lambda) d \lambda}{\mathrm{BW}} .
$$

The vegamag zero-point is defined as

$$
z p=+2.5 \log _{10}\left[\frac{\int f_{\lambda}(\lambda) R(\lambda) \lambda d \lambda}{\int R(\lambda) \lambda d \lambda}\right],
$$

while the flux at zero magnitude is calculated using the spectrum of Vega (corrected to have zero magnitude in all bands) such that

$$
f_{\nu} 0=\frac{\lambda_{\text {pivot }}^{2}}{c} \frac{\int f_{\lambda}(\lambda) R(\lambda) \lambda d \lambda}{\int R(\lambda) \lambda d \lambda},
$$

converted into Jansky, where $c$ is the speed of light. Table 4 shows these parameters calculated for the filters used in Table 3, where the BM12 naming is used. The table columns are defined as follows: (1) the filter name, (2) the mean photon wavelength, (3) the pivot wavelength, (4) the effective wavelength, (5) the nominal wavelength using the Rieke et al. (2008) definition, (6) the nominal wavelength using the Reach et al. (2005) definition, (7) the isophotal wavelength using the Rieke et al. (2008) definition, (8) the isophotal wavelength using the BM12 column definition, (9) the wavelength range, (10) the bandwidth, (11) the FWHM, (12) the filter response, (13) the zero-point for vegamag, (14) the corresponding flux density in $\operatorname{erg~s}^{-1} \mathrm{~cm}^{-2} \AA^{-1}$, and (15) the corresponding flux density in Jansky. 
Table 4

Filter Parameters

\begin{tabular}{|c|c|c|c|c|c|c|c|c|c|c|c|c|c|c|}
\hline Filter & $\begin{array}{c}\lambda_{\text {mean }} \\
(\mu \mathrm{m}) \\
(2)\end{array}$ & $\begin{array}{c}\lambda_{\text {pivot }} \\
(\mu \mathrm{m}) \\
(3)\end{array}$ & $\begin{array}{c}\lambda_{\text {eff }} \\
(\mu \mathrm{m}) \\
(4)\end{array}$ & $\begin{array}{c}\lambda_{n 1} \\
(\mu \mathrm{m}) \\
(5)\end{array}$ & $\begin{array}{c}\lambda_{n 2} \\
(\mu \mathrm{m}) \\
(6)\end{array}$ & $\begin{array}{c}\lambda_{i} \\
(\mu \mathrm{m}) \\
(7)\end{array}$ & $\begin{array}{c}\lambda_{i}(\mathrm{BM} 12) \\
(\mu \mathrm{m}) \\
(8)\end{array}$ & $\begin{array}{c}\lambda_{\text {range }} \\
(\mu \mathrm{m}) \\
(9)\end{array}$ & $\begin{array}{l}\mathrm{BW} \\
(\mu \mathrm{m}) \\
(10)\end{array}$ & $\begin{array}{c}\text { FWHM } \\
(\mu \mathrm{m}) \\
(11)\end{array}$ & $\begin{array}{c}\text { response } \\
\text { (12) }\end{array}$ & $\begin{array}{l}z p \\
(13)\end{array}$ & $\begin{array}{c}f_{\lambda}(z p) \\
\left(\mathrm{erg} \mathrm{s}^{-1} \mathrm{~cm}^{-2} \AA^{-1}\right) \\
(14)\end{array}$ & $\begin{array}{c}f_{\nu}(\operatorname{mag} 0) \\
(\mathrm{Jy}) \\
(15)\end{array}$ \\
\hline Johnson_U & 0.3618 & 0.3611 & 0.3694 & 0.3633 & 0.3603 & 0.3691 & 0.3719 & 0.1238 & 0.0581 & 0.0561 & 0.4693 & 20.9170 & $4.29723 \mathrm{E}-09$ & 1868.72 \\
\hline Johnson_B & 0.4410 & 0.4396 & 0.4390 & 0.4438 & 0.4382 & 0.3896 & 0.3911 & 0.1856 & 0.0992 & 0.1004 & 0.5345 & 20.4951 & $6.33795 \mathrm{E}-09$ & 4085.60 \\
\hline Johnson_V & 0.5524 & 0.5511 & 0.5476 & 0.5551 & 0.5499 & 0.5527 & 0.5510 & 0.2657 & 0.0871 & 0.0812 & 0.3275 & 21.1011 & 3.62701E-09 & 3674.73 \\
\hline Cousins_R & 0.6612 & 0.6582 & 0.6492 & 0.6674 & 0.6553 & 0.6545 & 0.6545 & 0.3322 & 0.1669 & 0.1671 & 0.5024 & 21.6781 & $2.13191 \mathrm{E}-09$ & 3080.98 \\
\hline Cousins_I & 0.8047 & 0.8034 & 0.7993 & 0.8074 & 0.8020 & 0.8006 & 0.8008 & 0.2163 & 0.1482 & 0.1523 & 0.6850 & 22.3469 & $1.15139 \mathrm{E}-09$ & 2478.76 \\
\hline Tycho_Bt & 0.4220 & 0.4212 & 0.4234 & 0.4237 & 0.4204 & 0.3944 & 0.3938 & 0.1548 & 0.0741 & 0.0739 & 0.4790 & 20.4332 & $6.70971 \mathrm{E}-09$ & 3970.61 \\
\hline Tycho_Vt & 0.5352 & 0.5335 & 0.5291 & 0.5389 & 0.5317 & 0.5359 & 0.5316 & 0.2263 & 0.1134 & 0.1116 & 0.5009 & 21.0088 & $3.94906 \mathrm{E}-09$ & 3748.93 \\
\hline Hipparcos_Hp & 0.5596 & 0.5508 & 0.5315 & 0.5780 & 0.5421 & 0.5467 & 0.5522 & 0.5772 & 0.2405 & 0.2237 & 0.4166 & 21.1107 & $3.59516 \mathrm{E}-09$ & 3638.04 \\
\hline 2MASS_J & 1.2411 & 1.2393 & 1.2321 & 1.2445 & 1.2376 & 1.2378 & 1.2377 & 0.3523 & 0.1628 & 0.2027 & 0.4611 & 23.7442 & $3.17931 \mathrm{E}-10$ & 1628.84 \\
\hline 2MASS_H & 1.6513 & 1.6495 & 1.6424 & 1.6551 & 1.6476 & 1.6467 & 1.6467 & 0.3826 & 0.2510 & 0.2610 & 0.6559 & 24.8385 & $1.16041 \mathrm{E}-10$ & 1053.12 \\
\hline 2MASS_Ks & 2.1656 & 2.1638 & 2.1558 & 2.1691 & 2.1621 & 2.1622 & 2.1625 & 0.4401 & 0.2622 & 0.2785 & 0.5951 & 25.8980 & $4.37335 \mathrm{E}-11$ & 683.04 \\
\hline SDSS_u & 0.3562 & 0.3556 & 0.3607 & 0.3572 & 0.3551 & 0.3642 & 0.3663 & 0.0983 & 0.0558 & 0.0582 & 0.0616 & 21.0632 & $3.75600 \mathrm{E}-09$ & 1584.71 \\
\hline SDSS_g & 0.4719 & 0.4702 & 0.4673 & 0.4751 & 0.4686 & 0.4730 & 0.4747 & 0.1963 & 0.1158 & 0.1263 & 0.2132 & 20.6442 & $5.52461 \mathrm{E}-09$ & 4075.09 \\
\hline SDSS_r & 0.6185 & 0.6176 & 0.6142 & 0.6204 & 0.6166 & 0.6169 & 0.6169 & 0.1723 & 0.1111 & 0.1150 & 0.3170 & 21.4800 & $2.55856 \mathrm{E}-09$ & 3254.86 \\
\hline SDSS_i & 0.7500 & 0.7490 & 0.7459 & 0.7519 & 0.7480 & 0.7498 & 0.7483 & 0.1844 & 0.1045 & 0.0683 & 0.2397 & 22.1124 & $1.42903 \mathrm{E}-09$ & 2674.11 \\
\hline SDSS_z & 0.8961 & 0.8947 & 0.8925 & 0.8992 & 0.8932 & 0.8933 & 0.8971 & 0.2781 & 0.1125 & 0.0994 & 0.0318 & 22.6604 & $8.62665 \mathrm{E}-10$ & 2303.28 \\
\hline DES_u & 0.3879 & 0.3859 & 0.3881 & 0.3970 & 0.3839 & 0.3774 & 0.3805 & 0.0711 & 0.0278 & 0.0256 & 0.0551 & 20.6479 & $5.50603 \mathrm{E}-09$ & 2735.17 \\
\hline DES_g & 0.4842 & 0.4820 & 0.4776 & 0.4890 & 0.4798 & 0.3811 & 0.3794 & 0.1663 & 0.1141 & 0.1299 & 0.2573 & 20.7091 & $5.20407 \mathrm{E}-09$ & 4033.18 \\
\hline DES_r & 0.6439 & 0.6423 & 0.6374 & 0.6470 & 0.6408 & 0.6379 & 0.6373 & 0.1901 & 0.1383 & 0.1484 & 0.3826 & 21.6052 & $2.27988 \mathrm{E}-09$ & 3137.37 \\
\hline DES_i & 0.7821 & 0.7807 & 0.7758 & 0.7848 & 0.7792 & 0.7626 & 0.7792 & 0.4647 & 0.1393 & 0.1482 & 0.1696 & 22.2523 & $1.25624 \mathrm{E}-09$ & 2553.68 \\
\hline DES_z & 0.9172 & 0.9158 & 0.9139 & 0.9196 & 0.9145 & 0.9574 & 0.9235 & 0.6876 & 0.1270 & 0.1479 & 0.1043 & 22.7104 & $8.23857 \mathrm{E}-10$ & 2304.99 \\
\hline DES_Y & 0.9877 & 0.9866 & 0.9830 & 0.9893 & 0.9855 & 0.9979 & 0.9898 & 0.1830 & 0.0680 & 0.0664 & 0.1642 & 22.9192 & $6.79718 \mathrm{E}-10$ & 2207.09 \\
\hline PS1_g & 0.4866 & 0.4849 & 0.4811 & 0.4900 & 0.4832 & 0.4866 & 0.4879 & 0.1707 & 0.1166 & 0.1256 & 0.3430 & 20.7241 & $5.13274 \mathrm{E}-09$ & 4025.81 \\
\hline PS1_r & 0.6215 & 0.6201 & 0.6156 & 0.6241 & 0.6188 & 0.6195 & 0.6200 & 0.1768 & 0.1318 & 0.1404 & 0.5121 & 21.4901 & $2.53499 \mathrm{E}-09$ & 3251.66 \\
\hline PS1_i & 0.7545 & 0.7535 & 0.7504 & 0.7564 & 0.7525 & 0.7525 & 0.7531 & 0.1659 & 0.1243 & 0.0698 & 0.6509 & 22.1328 & $1.40245 \mathrm{E}-09$ & 2656.00 \\
\hline PS1_z & 0.8679 & 0.8674 & 0.8669 & 0.8690 & 0.8669 & 0.8597 & 0.8662 & 0.1519 & 0.0966 & 0.1034 & 0.5596 & 22.5824 & $9.26899 \mathrm{E}-10$ & 2326.30 \\
\hline PS1_Y & 0.9633 & 0.9628 & 0.9614 & 0.9645 & 0.9622 & 0.9645 & 0.9621 & 0.1997 & 0.0616 & 0.0629 & 0.1893 & 22.8407 & $7.30650 \mathrm{E}-10$ & 2259.11 \\
\hline cfhtls_u & 0.3811 & 0.3803 & 0.3895 & 0.3829 & 0.3794 & 0.3810 & 0.3813 & 0.2267 & 0.0575 & 0.0654 & 0.1078 & 20.6447 & $5.52249 \mathrm{E}-09$ & 2663.85 \\
\hline cfhtls_g & 0.4862 & 0.4844 & 0.4803 & 0.4899 & 0.4826 & 0.4787 & 0.3774 & 0.2072 & 0.1322 & 0.1434 & 0.4045 & 20.7182 & $5.16084 \mathrm{E}-09$ & 4039.99 \\
\hline cfhtls_r & 0.6258 & 0.6248 & 0.6212 & 0.6279 & 0.6237 & 0.6241 & 0.6237 & 0.2001 & 0.1099 & 0.1219 & 0.3026 & 21.5171 & $2.47266 \mathrm{E}-09$ & 3219.42 \\
\hline cfhtls_i & 0.7690 & 0.7678 & 0.7638 & 0.7715 & 0.7666 & 0.7662 & 0.7667 & 0.2264 & 0.1221 & 0.1367 & 0.2594 & 22.1959 & $1.32325 \mathrm{E}-09$ & 2602.12 \\
\hline cfhtls_z & 0.8870 & 0.8859 & 0.8845 & 0.8894 & 0.8848 & 0.8840 & 0.8856 & 0.2270 & 0.0998 & 0.0936 & 0.1217 & 22.6349 & 8.83167E-10 & 2312.11 \\
\hline CFHT_12kx8k_B & 0.4407 & 0.4399 & 0.4400 & 0.4424 & 0.4390 & 0.3989 & 0.3879 & 0.2573 & 0.0619 & 0.0605 & 0.2405 & 20.4681 & $6.49800 \mathrm{E}-09$ & 4193.87 \\
\hline CFHT_12kx8k_R & 0.6621 & 0.6610 & 0.6578 & 0.6642 & 0.6600 & 0.6591 & 0.6581 & 0.1721 & 0.1077 & 0.1181 & 0.6257 & 21.7046 & $2.08038 \mathrm{E}-09$ & 3032.27 \\
\hline CFHT_12kx8k_I & 0.8183 & 0.8159 & 0.8096 & 0.8231 & 0.8136 & 0.8077 & 0.8107 & 0.2585 & 0.1921 & 0.2139 & 0.7409 & 22.3816 & $1.11520 \mathrm{E}-09$ & 2476.56 \\
\hline UKIRT_z & 0.8831 & 0.8826 & 0.8823 & 0.8840 & 0.8822 & 0.8820 & 0.8812 & 0.1403 & 0.0879 & 0.0926 & 0.1194 & 22.6261 & $8.90324 \mathrm{E}-10$ & 2313.54 \\
\hline UKIRT_Y & 1.0319 & 1.0315 & 1.0299 & 1.0329 & 1.0310 & 1.0307 & 1.0321 & 0.1569 & 0.1008 & 0.1034 & 0.1194 & 23.0663 & $5.93591 \mathrm{E}-10$ & 2106.53 \\
\hline UKIRT_J & 1.2511 & 1.2502 & 1.2462 & 1.2529 & 1.2492 & 1.2476 & 1.2490 & 0.2386 & 0.1475 & 0.1589 & 0.1321 & 23.7842 & $3.06416 \mathrm{E}-10$ & 1597.42 \\
\hline UKIRT_H & 1.6383 & 1.6360 & 1.6271 & 1.6430 & 1.6337 & 1.6313 & 1.6324 & 0.4649 & 0.2773 & 0.2918 & 0.1586 & 24.8055 & $1.19624 \mathrm{E}-10$ & 1067.96 \\
\hline UKIRT_K & 2.2085 & 2.2060 & 2.1950 & 2.2135 & 2.2035 & 2.2017 & 2.2032 & 0.5488 & 0.3276 & 0.3413 & 0.1422 & 25.9737 & $4.07863 \mathrm{E}-11$ & 662.09 \\
\hline LSST_u & 0.3671 & 0.3665 & 0.3743 & 0.3681 & 0.3660 & 0.3748 & 0.3724 & 0.0906 & 0.0547 & 0.0623 & 0.0829 & 20.8555 & 4.54799E-09 & 2038.03 \\
\hline LSST_g & 0.4827 & 0.4808 & 0.4768 & 0.4864 & 0.4789 & 0.4841 & 0.4859 & 0.1799 & 0.1333 & 0.1426 & 0.3027 & 20.7014 & $5.24138 \mathrm{E}-09$ & 4041.31 \\
\hline LSST_r & 0.6223 & 0.6210 & 0.6165 & 0.6250 & 0.6197 & 0.6206 & 0.6210 & 0.1685 & 0.1338 & 0.1343 & 0.3605 & 21.4946 & $2.52446 \mathrm{E}-09$ & 3247.24 \\
\hline LSST_i & 0.7546 & 0.7537 & 0.7506 & 0.7565 & 0.7527 & 0.7527 & 0.7533 & 0.1565 & 0.1209 & 0.0680 & 0.3490 & 22.1336 & 1.40133E-09 & 2655.04 \\
\hline LSST_z & 0.8691 & 0.8686 & 0.8680 & 0.8702 & 0.8680 & 0.8622 & 0.8681 & 0.1350 & 0.0994 & 0.1022 & 0.3269 & 22.5857 & $9.24140 \mathrm{E}-10$ & 2325.47 \\
\hline LSST_y & 0.9710 & 0.9705 & 0.9688 & 0.9722 & 0.9699 & 0.9686 & 0.9717 & 0.1828 & 0.0814 & 0.0857 & 0.1259 & 22.8627 & $7.16026 \mathrm{E}-10$ & 2249.34 \\
\hline
\end{tabular}


Table 4

(Continued)

\begin{tabular}{|c|c|c|c|c|c|c|c|c|c|c|c|c|c|c|}
\hline Filter & $\begin{array}{c}\lambda_{\text {mean }} \\
(\mu \mathrm{m}) \\
(2)\end{array}$ & $\begin{array}{l}\lambda_{\text {pivot }} \\
(\mu \mathrm{m}) \\
(3)\end{array}$ & $\begin{array}{l}\lambda_{\text {eff }} \\
(\mu \mathrm{m}) \\
(4)\end{array}$ & $\begin{array}{l}\lambda_{n 1} \\
(\mu \mathrm{m}) \\
(5)\end{array}$ & $\begin{array}{l}\lambda_{n 2} \\
(\mu \mathrm{m}) \\
(6)\end{array}$ & $\begin{array}{c}\lambda_{i} \\
(\mu \mathrm{m}) \\
(7)\end{array}$ & $\begin{array}{l}\lambda_{i}(\mathrm{BM} 12) \\
\quad(\mu \mathrm{m}) \\
(8)\end{array}$ & $\begin{array}{c}\lambda_{\text {range }} \\
(\mu \mathrm{m}) \\
(9)\end{array}$ & $\begin{array}{l}\mathrm{BW} \\
(\mu \mathrm{m}) \\
(10)\end{array}$ & $\begin{array}{c}\text { FWHM } \\
(\mu \mathrm{m}) \\
(11)\end{array}$ & $\begin{array}{c}\text { response } \\
\text { (12) }\end{array}$ & $\begin{array}{l}z p \\
(13)\end{array}$ & $\begin{array}{c}\underset{f_{\lambda}(z p)}{\left(\mathrm{erg} \mathrm{s}^{-1} \mathrm{~cm}^{-2} \AA^{-1}\right)} \\
(14)\end{array}$ & $\begin{array}{c}f_{\nu}(\operatorname{mag} 0) \\
(\mathrm{Jy}) \\
(15)\end{array}$ \\
\hline Bessell_Murphy_U & 0.3604 & 0.3597 & 0.3674 & 0.3617 & 0.3591 & 0.3674 & 0.3680 & 0.1098 & 0.0621 & 0.0628 & 0.5656 & 20.9560 & $4.14586 \mathrm{E}-09$ & 1789.43 \\
\hline Bessell_Murphy_B & 0.4391 & 0.4378 & 0.4369 & 0.4420 & 0.4364 & 0.4601 & 0.3914 & 0.1796 & 0.0916 & 0.0894 & 0.5098 & 20.4800 & $6.42715 \mathrm{E}-09$ & 4108.32 \\
\hline Bessell_Murphy_V & 0.5501 & 0.5489 & 0.5457 & 0.5525 & 0.5477 & 0.5513 & 0.5483 & 0.2495 & 0.0875 & 0.0836 & 0.3507 & 21.0884 & 3.66982E-09 & 3687.87 \\
\hline Bessell_Murphy_R & 0.6554 & 0.6524 & 0.6436 & 0.6616 & 0.6495 & 0.6483 & 0.6483 & 0.3393 & 0.1485 & 0.1447 & 0.4375 & 21.6485 & $2.19072 \mathrm{E}-09$ & 3110.41 \\
\hline Bessell_Murphy_I & 0.7996 & 0.7984 & 0.7943 & 0.8023 & 0.7971 & 0.7956 & 0.7960 & 0.1996 & 0.1427 & 0.1498 & 0.7147 & 22.3268 & $1.17294 \mathrm{E}-09$ & 2493.70 \\
\hline Bessell_Murphy_Bt & 0.4198 & 0.4190 & 0.4215 & 0.4214 & 0.4182 & 0.3928 & 0.3941 & 0.1447 & 0.0719 & 0.0719 & 0.4966 & 20.4308 & $6.72504 \mathrm{E}-09$ & 3938.09 \\
\hline Bessell_Murphy_Vt & 0.5315 & 0.5300 & 0.5266 & 0.5345 & 0.5285 & 0.5283 & 0.5295 & 0.2096 & 0.0993 & 0.0963 & 0.4737 & 20.9915 & 4.01224E-09 & 3759.32 \\
\hline Bessell_Murphy_Hp & 0.5429 & 0.5349 & 0.5188 & 0.5595 & 0.5271 & 0.5694 & 0.5352 & 0.5289 & 0.2269 & 0.2117 & 0.4290 & 21.0316 & 3.86674E-09 & 3691.00 \\
\hline Bessell_88_J & 1.2369 & 1.2347 & 1.2258 & 1.2412 & 1.2325 & 1.2322 & 1.2326 & 0.3593 & 0.2029 & 0.2066 & 0.5308 & 23.7318 & $3.21587 \mathrm{E}-10$ & 1635.23 \\
\hline Bessell_88_H & 1.6472 & 1.6450 & 1.6365 & 1.6517 & 1.6428 & 1.6406 & 1.6414 & 0.3393 & 0.2845 & 0.2984 & 0.8301 & 24.8263 & $1.17350 \mathrm{E}-10$ & 1059.24 \\
\hline Bessell_88_K & 2.1683 & 2.1663 & 2.1574 & 2.1721 & 2.1644 & 2.1596 & 2.1637 & 0.3593 & 0.2837 & 0.3048 & 0.7738 & 25.9019 & $4.35755 \mathrm{E}-11$ & 682.12 \\
\hline Bessell_88_L & 3.4838 & 3.4797 & 3.4602 & 3.4919 & 3.4756 & 3.4728 & 3.4733 & 0.7186 & 0.4583 & 0.5103 & 0.5611 & 27.8367 & $7.33364 \mathrm{E}-12$ & 296.20 \\
\hline Bessell_88_Lprime & 3.8285 & 3.8247 & 3.8063 & 3.8362 & 3.8208 & 3.8154 & 3.8182 & 0.6786 & 0.5339 & 0.5880 & 0.7632 & 28.2347 & $5.08305 \mathrm{E}-12$ & 248.03 \\
\hline Bessell_88_M & 4.7369 & 4.7347 & 4.7250 & 4.7411 & 4.7326 & 4.7241 & 4.7325 & 0.5589 & 0.3498 & 0.2044 & 0.3127 & 29.1335 & $2.22134 \mathrm{E}-12$ & 166.11 \\
\hline GALEX_FUVV & 0.1539 & 0.1535 & 0.1549 & 0.1546 & 0.1532 & 0.1464 & 0.1469 & 0.0453 & 0.0255 & 0.0228 & 0.0106 & 20.4239 & $6.76768 \mathrm{E}-09$ & 531.97 \\
\hline GALEX_NUV & 0.2316 & 0.2301 & 0.2304 & 0.2345 & 0.2286 & 0.2272 & 0.2269 & 0.1185 & 0.0730 & 0.0796 & 0.0193 & 20.8469 & 4.58413E-09 & 809.45 \\
\hline WISE_1 & 3.4003 & 3.3897 & 3.3387 & 3.4204 & 3.3792 & 3.3687 & 3.3722 & 1.3441 & 0.6628 & 0.6358 & 0.4930 & 27.7140 & $8.21074 \mathrm{E}-12$ & 314.69 \\
\hline WISE_2 & 4.6520 & 4.6406 & 4.5870 & 4.6746 & 4.6293 & 4.6204 & 4.6199 & 1.4623 & 1.0423 & 1.1073 & 0.7128 & 29.0322 & $2.43841 \mathrm{E}-12$ & 175.16 \\
\hline WISE_3 & 12.8114 & 12.5705 & 11.3086 & 13.2371 & 12.3341 & 11.6601 & 12.0626 & 18.3366 & 5.5114 & 6.2771 & 0.3003 & 33.1194 & $5.65225 \mathrm{E}-14$ & 29.79 \\
\hline WISE_4 & 22.3753 & 22.3142 & 22.0230 & 22.5013 & 22.2533 & 22.1724 & 22.1950 & 8.8919 & 4.1023 & 3.6087 & 0.4613 & 35.7525 & $5.00014 \mathrm{E}-15$ & 8.30 \\
\hline IRAS12 & 11.5406 & 11.3562 & 10.4650 & 11.8905 & 11.1747 & 10.8564 & 10.9983 & 7.4850 & 5.9671 & 6.9307 & 0.7971 & 32.7211 & $8.15757 \mathrm{E}-14$ & 35.09 \\
\hline IRAS25 & 23.8767 & 23.6079 & 22.2580 & 24.3900 & 23.3421 & 23.1021 & 23.0659 & 14.9700 & 10.0234 & 11.2592 & 0.6688 & 35.9191 & $4.28907 \mathrm{E}-15$ & 7.97 \\
\hline IRAS60 & 61.4459 & 60.3699 & 54.5695 & 63.3790 & 59.3127 & 52.8094 & 58.0207 & 53.8919 & 30.4317 & 32.7622 & 0.5646 & 39.9327 & $1.06394 \mathrm{E}-16$ & 1.29 \\
\hline IRAS100 & 101.9433 & 101.1267 & 96.9972 & 103.5466 & 100.3167 & 99.6179 & 99.4636 & 69.8599 & 33.2387 & 32.2401 & 0.4754 & 42.2860 & $1.21782 \mathrm{E}-17$ & 0.42 \\
\hline IRAC_3.6 & 3.5573 & 3.5508 & 3.5204 & 3.5701 & 3.5443 & 3.5375 & 3.5400 & 0.8893 & 0.6836 & 0.7432 & 0.3639 & 27.9174 & $6.80809 \mathrm{E}-12$ & 286.32 \\
\hline IRAC_4.5 & 4.5049 & 4.4960 & 4.4543 & 4.5228 & 4.4870 & 4.4785 & 4.4786 & 1.3434 & 0.8650 & 1.0097 & 0.3529 & 28.9037 & $2.74485 \mathrm{E}-12$ & 185.07 \\
\hline IRAC_5.8 & 5.7386 & 5.7245 & 5.6564 & 5.7664 & 5.7104 & 5.6999 & 5.6972 & 1.6151 & 1.2562 & 1.3912 & 0.1105 & 29.9163 & $1.08012 \mathrm{E}-12$ & 118.07 \\
\hline IRAC_8.0 & 7.9274 & 7.8842 & 7.6741 & 8.0118 & 7.8413 & 7.7845 & 7.8010 & 3.3582 & 2.5292 & 2.8311 & 0.2365 & 31.2516 & $3.15764 \mathrm{E}-13$ & 65.47 \\
\hline IRS_16 & 16.0478 & 15.9222 & 15.4020 & 16.3590 & 15.7975 & 15.7463 & 15.7090 & 22.8531 & 4.7674 & 5.4763 & 0.6163 & 34.2569 & $1.98254 \mathrm{E}-14$ & 16.77 \\
\hline IRS_22 & 22.6224 & 22.4704 & 21.7563 & 22.9355 & 22.3193 & 22.2729 & 22.1796 & 18.7995 & 7.0115 & 7.3067 & 0.8122 & 35.7499 & $5.01246 \mathrm{E}-15$ & 8.44 \\
\hline MIPS_24 & 23.8436 & 23.7592 & 23.3583 & 24.0181 & 23.6750 & 23.6079 & 23.5923 & 12.6666 & 5.2969 & 5.3248 & 0.4181 & 36.0180 & $3.91555 \mathrm{E}-15$ & 7.37 \\
\hline MIPS_70 & 72.5564 & 71.9861 & 69.3644 & 73.7885 & 71.4202 & 70.8090 & 70.9157 & 60.4363 & 21.3011 & 18.9838 & 0.3527 & 40.8081 & $4.75089 \mathrm{E}-17$ & 0.82 \\
\hline MIPS_160 & 156.9627 & 156.4274 & 153.6888 & 158.0193 & 155.8939 & 155.4756 & 155.3366 & 92.3398 & 35.7629 & 34.5528 & 0.3872 & 44.2365 & $2.02017 \mathrm{E}-18$ & 0.16 \\
\hline ACS_F330W & 0.3522 & 0.3521 & 0.3523 & 0.3525 & 0.3520 & 0.3485 & 0.3593 & 0.0474 & 0.0261 & 0.0272 & 0.0454 & 21.2385 & $3.19592 \mathrm{E}-09$ & 1321.74 \\
\hline ACS_F410W & 0.4069 & 0.4064 & 0.4096 & 0.4078 & 0.4059 & 0.4535 & 0.3954 & 0.0890 & 0.0522 & 0.0543 & 0.2040 & 20.4195 & $6.79505 \mathrm{E}-09$ & 3743.07 \\
\hline ACS_F435W & 0.4338 & 0.4328 & 0.4341 & 0.4358 & 0.4318 & 0.3892 & 0.3940 & 0.1330 & 0.0863 & 0.0935 & 0.2387 & 20.4608 & $6.54181 \mathrm{E}-09$ & 4087.92 \\
\hline ACS_F475W & 0.4766 & 0.4747 & 0.4710 & 0.4802 & 0.4728 & 0.4812 & 0.4805 & 0.1781 & 0.1359 & 0.1437 & 0.2807 & 20.6676 & 5.40703E-09 & 4064.13 \\
\hline ACS_F555W & 0.5373 & 0.5361 & 0.5333 & 0.5398 & 0.5349 & 0.5339 & 0.5329 & 0.1715 & 0.1125 & 0.1240 & 0.2408 & 21.0244 & $3.89261 \mathrm{E}-09$ & 3731.78 \\
\hline ACS_F606W & 0.5960 & 0.5922 & 0.5812 & 0.6035 & 0.5883 & 0.5895 & 0.5917 & 0.2601 & 0.1996 & 0.2323 & 0.3588 & 21.3334 & $2.92845 \mathrm{E}-09$ & 3425.32 \\
\hline ACS_F625W & 0.6325 & 0.6312 & 0.6267 & 0.6352 & 0.6298 & 0.6295 & 0.6307 & 0.1715 & 0.1308 & 0.1416 & 0.3372 & 21.5483 & $2.40256 \mathrm{E}-09$ & 3192.67 \\
\hline ACS_F775W & 0.7707 & 0.7694 & 0.7654 & 0.7732 & 0.7682 & 0.7682 & 0.7680 & 0.1874 & 0.1320 & 0.1511 & 0.3011 & 22.2032 & $1.31439 \mathrm{E}-09$ & 2595.74 \\
\hline ACS_F814W & 0.8086 & 0.8059 & 0.7987 & 0.8142 & 0.8031 & 0.7990 & 0.8000 & 0.2891 & 0.1739 & 0.1856 & 0.2654 & 22.3391 & $1.15969 \mathrm{E}-09$ & 2512.22 \\
\hline ACS_F850LP & 0.9030 & 0.9016 & 0.8994 & 0.9060 & 0.9001 & 0.9006 & 0.9006 & 0.2534 & 0.1247 & 0.1210 & 0.1230 & 22.6773 & $8.49320 \mathrm{E}-10$ & 2302.73 \\
\hline WFC3_F218W & 0.2233 & 0.2229 & 0.2233 & 0.2242 & 0.2224 & 0.3635 & 0.3786 & 0.0570 & 0.0330 & 0.0340 & 0.0239 & 20.8017 & 4.77894E-09 & 791.70 \\
\hline WFC3_F225W & 0.2379 & 0.2372 & 0.2374 & 0.2392 & 0.2365 & 0.2350 & 0.2335 & 0.0975 & 0.0467 & 0.0470 & 0.0404 & 20.9087 & $4.33036 \mathrm{E}-09$ & 812.62 \\
\hline WFC3_F336W & 0.3359 & 0.3355 & 0.3359 & 0.3366 & 0.3351 & 0.3371 & 0.3523 & 0.0714 & 0.0512 & 0.0550 & 0.1403 & 21.1944 & $3.32855 \mathrm{E}-09$ & 1249.55 \\
\hline
\end{tabular}


Table 4

(Continued)

\begin{tabular}{|c|c|c|c|c|c|c|c|c|c|c|c|c|c|c|}
\hline Filter & $\begin{array}{l}\lambda_{\text {mean }} \\
(\mu \mathrm{m}) \\
(2)\end{array}$ & $\begin{array}{l}\lambda_{\text {pivot }} \\
(\mu \mathrm{m}) \\
(3)\end{array}$ & $\begin{array}{l}\lambda_{\text {eff }} \\
(\mu \mathrm{m}) \\
(4)\end{array}$ & $\begin{array}{l}\lambda_{n 1} \\
(\mu \mathrm{m}) \\
(5)\end{array}$ & $\begin{array}{l}\lambda_{n 2} \\
(\mu \mathrm{m}) \\
(6)\end{array}$ & $\begin{array}{c}\lambda_{i} \\
(\mu \mathrm{m}) \\
(7)\end{array}$ & $\begin{array}{l}\lambda_{i}(\mathrm{BM} 12) \\
\quad(\mu \mathrm{m}) \\
(8)\end{array}$ & $\begin{array}{c}\lambda_{\text {range }} \\
(\mu \mathrm{m}) \\
(9)\end{array}$ & $\begin{array}{l}\mathrm{BW} \\
(\mu \mathrm{m}) \\
(10)\end{array}$ & $\begin{array}{c}\text { FWHM } \\
(\mu \mathrm{m}) \\
(11)\end{array}$ & $\begin{array}{l}\text { response } \\
\text { (12) }\end{array}$ & $\begin{array}{l}z p \\
(13)\end{array}$ & $\begin{array}{c}f_{\lambda}(z p) \\
\left(\mathrm{erg} \mathrm{s}^{-1} \mathrm{~cm}^{-2} \AA^{-1}\right) \\
(14)\end{array}$ & $\begin{array}{c}f_{\nu}(\operatorname{mag} 0) \\
(\mathrm{Jy}) \\
(15)\end{array}$ \\
\hline WFC3_F390W & 0.3935 & 0.3924 & 0.4023 & 0.3956 & 0.3914 & 0.4522 & 0.3885 & 0.1247 & 0.0893 & 0.0948 & 0.1763 & 20.5639 & $5.94869 \mathrm{E}-09$ & 3055.96 \\
\hline WFC3_F438W & 0.4331 & 0.4326 & 0.4324 & 0.4340 & 0.4322 & 0.3884 & 0.3983 & 0.0849 & 0.0614 & 0.0673 & 0.1745 & 20.4103 & $6.85275 \mathrm{E}-09$ & 4278.69 \\
\hline WFC3_F475W & 0.4792 & 0.4774 & 0.4734 & 0.4829 & 0.4755 & 0.4763 & 0.4835 & 0.1671 & 0.1342 & 0.1481 & 0.2154 & 20.6805 & $5.34326 \mathrm{E}-09$ & 4061.51 \\
\hline WFC3_F555W & 0.5335 & 0.5308 & 0.5238 & 0.5389 & 0.5282 & 0.5309 & 0.5293 & 0.2846 & 0.1564 & 0.1579 & 0.1558 & 20.9843 & 4.03892E-09 & 3796.05 \\
\hline WFC3_F606W & 0.5925 & 0.5887 & 0.5783 & 0.5999 & 0.5850 & 0.5917 & 0.5883 & 0.2561 & 0.2184 & 0.2298 & 0.2472 & 21.3170 & $2.97294 \mathrm{E}-09$ & 3437.24 \\
\hline WFC3_F625W & 0.6258 & 0.6241 & 0.6188 & 0.6291 & 0.6225 & 0.6222 & 0.6241 & 0.1762 & 0.1460 & 0.1573 & 0.2323 & 21.5086 & 2.49207E-09 & 3238.08 \\
\hline WFC3_F775W & 0.7660 & 0.7648 & 0.7611 & 0.7684 & 0.7637 & 0.7642 & 0.7636 & 0.1752 & 0.1170 & 0.1455 & 0.1555 & 22.1828 & $1.33934 \mathrm{E}-09$ & 2613.48 \\
\hline WFC3_F814W & 0.8058 & 0.8030 & 0.7955 & 0.8117 & 0.8001 & 0.7956 & 0.7962 & 0.2746 & 0.1540 & 0.1518 & 0.1303 & 22.3259 & 1.17397E-09 & 2524.73 \\
\hline WFC3_F098m & 0.9877 & 0.9864 & 0.9828 & 0.9903 & 0.9852 & 0.9872 & 0.9842 & 0.2085 & 0.1570 & 0.1694 & 0.3524 & 22.9160 & $6.81702 \mathrm{E}-10$ & 2212.63 \\
\hline WFC3_F105W & 1.0585 & 1.0551 & 1.0432 & 1.0652 & 1.0517 & 1.0560 & 1.0530 & 0.3290 & 0.2650 & 0.2917 & 0.4149 & 23.1459 & $5.51635 \mathrm{E}-10$ & 2048.30 \\
\hline WFC3_F125W & 1.2516 & 1.2486 & 1.2365 & 1.2576 & 1.2456 & 1.2455 & 1.2436 & 0.3427 & 0.2845 & 0.3005 & 0.4553 & 23.7673 & $3.11218 \mathrm{E}-10$ & 1618.43 \\
\hline WFC3_F140W & 1.3969 & 1.3922 & 1.3733 & 1.4061 & 1.3875 & 1.3804 & 1.3840 & 0.4438 & 0.3842 & 0.3941 & 0.4807 & 24.1788 & $2.13053 \mathrm{E}-10$ & 1377.41 \\
\hline WFC3_F160W & 1.5392 & 1.5370 & 1.5279 & 1.5436 & 1.5348 & 1.5322 & 1.5341 & 0.3302 & 0.2682 & 0.2874 & 0.4481 & 24.5692 & $1.48698 \mathrm{E}-10$ & 1171.80 \\
\hline WFPC2_F218W & 0.2214 & 0.2207 & 0.2205 & 0.2228 & 0.2200 & 0.3769 & 0.3781 & 0.0510 & 0.0451 & 0.0436 & 0.0026 & 20.7838 & $4.85820 \mathrm{E}-09$ & 789.30 \\
\hline WFPC2_F300W & 0.3013 & 0.2992 & 0.3039 & 0.3066 & 0.2972 & 0.2382 & 0.5499 & 0.1473 & 0.0857 & 0.0867 & 0.0116 & 21.0950 & $3.64742 \mathrm{E}-09$ & 1089.44 \\
\hline WFPC2_F450W & 0.4574 & 0.4556 & 0.4547 & 0.4608 & 0.4539 & 0.3830 & 0.3831 & 0.1730 & 0.0875 & 0.1078 & 0.0439 & 20.5907 & $5.80384 \mathrm{E}-09$ & 4019.36 \\
\hline WFPC2_F555W & 0.5468 & 0.5442 & 0.5373 & 0.5519 & 0.5417 & 0.5437 & 0.5416 & 0.2717 & 0.1456 & 0.1558 & 0.0604 & 21.0621 & $3.75986 \mathrm{E}-09$ & 3714.54 \\
\hline WFPC2_F606W & 0.6035 & 0.6001 & 0.5902 & 0.6101 & 0.5967 & 0.6009 & 0.5993 & 0.2677 & 0.1888 & 0.2002 & 0.1017 & 21.3763 & $2.81505 \mathrm{E}-09$ & 3381.55 \\
\hline WFPC2_F702W & 0.6945 & 0.6919 & 0.6841 & 0.6997 & 0.6893 & 0.6885 & 0.6884 & 0.2613 & 0.1666 & 0.1875 & 0.0920 & 21.8481 & 1.82293E-09 & 2910.78 \\
\hline WFPC2_F814W & 0.8029 & 0.8002 & 0.7930 & 0.8087 & 0.7974 & 0.7930 & 0.7938 & 0.2859 & 0.1485 & 0.1455 & 0.0548 & 22.3159 & $1.18478 \mathrm{E}-09$ & 2530.29 \\
\hline NIC2_F110W & 1.1353 & 1.1235 & 1.0840 & 1.1575 & 1.1119 & 1.1232 & 1.1204 & 0.6564 & 0.4284 & 0.5272 & 0.1111 & 23.3646 & $4.50974 \mathrm{E}-10$ & 1898.82 \\
\hline NIC2_F160W & 1.6074 & 1.6030 & 1.5859 & 1.6159 & 1.5987 & 1.5932 & 1.5959 & 0.5042 & 0.3416 & 0.4013 & 0.1681 & 24.7184 & $1.29607 \mathrm{E}-10$ & 1110.96 \\
\hline NIC3_F110W & 1.1326 & 1.1200 & 1.0788 & 1.1561 & 1.1076 & 1.1142 & 1.1156 & 0.6568 & 0.4253 & 0.5883 & 0.1013 & 23.3547 & $4.55131 \mathrm{E}-10$ & 1904.49 \\
\hline NIC3_F160W & 1.6085 & 1.6042 & 1.5872 & 1.6169 & 1.5999 & 1.5965 & 1.5968 & 0.4967 & 0.3394 & 0.3987 & 0.1595 & 24.7213 & $1.29270 \mathrm{E}-10$ & 1109.63 \\
\hline NIRCAM_F070W & 0.7066 & 0.7046 & 0.6991 & 0.7114 & 0.7027 & 0.7053 & 0.7048 & 0.1896 & 0.1325 & 0.1600 & 0.1732 & 21.9113 & $1.71987 \mathrm{E}-09$ & 2848.45 \\
\hline NIRCAM_F090W & 0.9047 & 0.9025 & 0.8988 & 0.9093 & 0.9003 & 0.9039 & 0.9039 & 0.2383 & 0.1943 & 0.2101 & 0.2599 & 22.6730 & $8.52676 \mathrm{E}-10$ & 2316.81 \\
\hline NIRCAM_F115W & 1.1570 & 1.1543 & 1.1435 & 1.1624 & 1.1515 & 1.1526 & 1.1523 & 0.3155 & 0.2246 & 0.2683 & 0.2792 & 23.4729 & $4.08160 \mathrm{E}-10$ & 1813.92 \\
\hline NIRCAM_F140M & 1.4060 & 1.4053 & 1.4024 & 1.4074 & 1.4046 & 1.4064 & 1.4038 & 0.2186 & 0.1425 & 0.1478 & 0.2786 & 24.2262 & $2.03956 \mathrm{E}-10$ & 1343.59 \\
\hline NIRCAM_F150W & 1.5040 & 1.5007 & 1.4873 & 1.5104 & 1.4975 & 1.4974 & 1.4961 & 0.4093 & 0.3180 & 0.3371 & 0.3510 & 24.4711 & $1.62767 \mathrm{E}-10$ & 1222.81 \\
\hline NIRCAM_F150W2 & 1.7039 & 1.6588 & 1.4796 & 1.7864 & 1.6150 & 1.6383 & 1.5932 & 1.6908 & 1.1753 & 1.3255 & 0.3248 & 24.7096 & $1.30667 \mathrm{E}-10$ & 1199.33 \\
\hline NIRCAM_F162M & 1.6281 & 1.6272 & 1.6244 & 1.6297 & 1.6264 & 1.6255 & 1.6263 & 0.2522 & 0.1683 & 0.1714 & 0.2940 & 24.7932 & $1.20984 \mathrm{E}-10$ & 1068.60 \\
\hline NIRCAM_F164N & 1.6446 & 1.6445 & 1.6446 & 1.6446 & 1.6445 & 1.6386 & 1.6441 & 0.0602 & 0.0200 & 0.0179 & 0.1433 & 24.8561 & $1.14173 \mathrm{E}-10$ & 1029.98 \\
\hline NIRCAM_F182M & 1.8466 & 1.8452 & 1.8389 & 1.8494 & 1.8437 & 1.8423 & 1.8435 & 0.3335 & 0.2377 & 0.2460 & 0.3430 & 25.2723 & $7.78176 \mathrm{E}-11$ & 883.75 \\
\hline NIRCAM_F187N & 1.8739 & 1.8739 & 1.8737 & 1.8740 & 1.8739 & 1.8698 & 1.8731 & 0.0651 & 0.0237 & 0.0211 & 0.1650 & 25.3720 & $7.09931 \mathrm{E}-11$ & 831.55 \\
\hline NIRCAM_F200W & 1.9934 & 1.9886 & 1.9681 & 2.0028 & 1.9839 & 1.9803 & 1.9828 & 0.5611 & 0.4566 & 0.4717 & 0.4016 & 25.5530 & $6.00903 \mathrm{E}-11$ & 792.68 \\
\hline NIRCAM_F200W & 1.9934 & 1.9886 & 1.9681 & 2.0028 & 1.9839 & 1.9803 & 1.9828 & 0.5611 & 0.4566 & 0.4717 & 0.4016 & 25.5530 & $6.00903 \mathrm{E}-11$ & 792.68 \\
\hline NIRCAM_F210M & 2.0964 & 2.0955 & 2.0908 & 2.0982 & 2.0945 & 2.0956 & 2.0946 & 0.2955 & 0.2065 & 0.2090 & 0.3391 & 25.7709 & $4.91626 \mathrm{E}-11$ & 720.06 \\
\hline NIRCAM_F250M & 2.5038 & 2.5032 & 2.5006 & 2.5049 & 2.5027 & 2.5046 & 2.5026 & 0.2410 & 0.1800 & 0.1826 & 0.2979 & 26.4931 & $2.52780 \mathrm{E}-11$ & 528.36 \\
\hline NIRCAM_F277W & 2.7694 & 2.7618 & 2.7280 & 2.7845 & 2.7542 & 2.7476 & 2.7471 & 0.8977 & 0.6828 & 0.7111 & 0.3129 & 26.8793 & $1.77123 \mathrm{E}-11$ & 450.64 \\
\hline NIRCAM_F300M & 2.9908 & 2.9892 & 2.9819 & 2.9941 & 2.9875 & 2.9862 & 2.9852 & 0.5259 & 0.3153 & 0.3264 & 0.2353 & 27.2152 & $1.29998 \mathrm{E}-11$ & 387.46 \\
\hline NIRCAM_F322W2 & 3.2668 & 3.2320 & 3.0736 & 3.3335 & 3.1976 & 3.1789 & 3.1733 & 1.7937 & 1.3563 & 1.5827 & 0.3959 & 27.4646 & $1.03319 \mathrm{E}-11$ & 359.99 \\
\hline NIRCAM_F323N & 3.2370 & 3.2369 & 3.2368 & 3.2370 & 3.2369 & 3.2368 & 3.2375 & 0.0836 & 0.0385 & 0.0386 & 0.1466 & 27.5412 & $9.62799 \mathrm{E}-12$ & 336.50 \\
\hline NIRCAM_F335M & 3.3640 & 3.3621 & 3.3539 & 3.3676 & 3.3603 & 3.3592 & 3.3582 & 0.6118 & 0.3520 & 0.3608 & 0.2626 & 27.6991 & $8.32451 \mathrm{E}-12$ & 313.89 \\
\hline NIRCAM_F356W & 3.5768 & 3.5684 & 3.5287 & 3.5935 & 3.5600 & 3.5553 & 3.5532 & 1.0833 & 0.7811 & 0.8407 & 0.3766 & 27.9334 & $6.70861 \mathrm{E}-12$ & 284.94 \\
\hline NIRCAM_F405N & 4.0517 & 4.0517 & 4.0516 & 4.0517 & 4.0516 & 4.0475 & 4.0515 & 0.1108 & 0.0455 & 0.0460 & 0.1736 & 28.5039 & $3.96684 \mathrm{E}-12$ & 217.21 \\
\hline NIRCAM_F410M & 4.0844 & 4.0822 & 4.0723 & 4.0887 & 4.0801 & 4.0791 & 4.0790 & 0.7083 & 0.4379 & 0.4375 & 0.3096 & 28.5111 & $3.94060 \mathrm{E}-12$ & 219.05 \\
\hline
\end{tabular}


Table 4

\begin{tabular}{|c|c|c|c|c|c|c|c|c|c|c|c|c|c|c|}
\hline Filter & $\begin{array}{l}\lambda_{\text {mean }} \\
(\mu \mathrm{m}) \\
(2)\end{array}$ & $\begin{array}{l}\lambda_{\text {pivot }} \\
(\mu \mathrm{m}) \\
(3)\end{array}$ & $\begin{array}{l}\lambda_{\text {eff }} \\
(\mu \mathrm{m}) \\
(4)\end{array}$ & $\begin{array}{l}\lambda_{n 1} \\
(\mu \mathrm{m}) \\
(5)\end{array}$ & $\begin{array}{l}\lambda_{n 2} \\
(\mu \mathrm{m}) \\
(6)\end{array}$ & $\begin{array}{c}\lambda_{i} \\
(\mu \mathrm{m}) \\
(7)\end{array}$ & $\begin{array}{l}\lambda_{i}(\mathrm{BM} 12) \\
\quad(\mu \mathrm{m}) \\
\quad(8)\end{array}$ & $\begin{array}{l}\lambda_{\text {range }} \\
(\mu \mathrm{m}) \\
(9)\end{array}$ & $\begin{array}{l}\mathrm{BW} \\
(\mu \mathrm{m}) \\
(10)\end{array}$ & $\begin{array}{c}\text { FWHM } \\
(\mu \mathrm{m}) \\
(11)\end{array}$ & $\begin{array}{l}\text { response } \\
\text { (12) }\end{array}$ & $\begin{array}{l}z p \\
(13)\end{array}$ & $\begin{array}{c}\left.\mathrm{erg} \mathrm{s}^{-1} \underset{\mathrm{\lambda}^{\lambda}(z p)}{\mathrm{cm}^{-2}} \AA^{-1}\right) \\
(14)\end{array}$ & $\begin{array}{l}f_{\nu}(\operatorname{mag} 0) \\
(\mathrm{Jy}) \\
(15)\end{array}$ \\
\hline NIRCAM_F430M & 4.2818 & 4.2813 & 4.2785 & 4.2829 & 4.2807 & 4.2806 & 4.2811 & 0.3664 & 0.2277 & 0.2312 & 0.3109 & 28.7127 & $3.27266 \mathrm{E}-12$ & 200.09 \\
\hline NIRCAM_F444W & 4.4157 & 4.4040 & 4.3496 & 4.4392 & 4.3923 & 4.4440 & 4.3830 & 2.6004 & 1.0316 & 1.1055 & 0.2042 & 28.8119 & $2.98700 \mathrm{E}-12$ & 193.24 \\
\hline NIRCAM_F460M & 4.6293 & 4.6285 & 4.6229 & 4.6308 & 4.6276 & 4.6253 & 4.6192 & 0.4007 & 0.2288 & 0.2323 & 0.2433 & 29.0433 & $2.41358 \mathrm{E}-12$ & 172.47 \\
\hline NIRCAM_F466N & 4.6545 & 4.6544 & 4.6540 & 4.6545 & 4.6544 & 4.6496 & 4.6544 & 0.1311 & 0.0536 & 0.0520 & 0.1347 & 29.0995 & $2.29198 \mathrm{E}-12$ & 165.62 \\
\hline NIRCAM_F470N & 4.7078 & 4.7078 & 4.7078 & 4.7079 & 4.7078 & 4.7054 & 4.7077 & 0.1196 & 0.0510 & 0.0495 & 0.1299 & 29.1127 & $2.26424 \mathrm{E}-12$ & 167.39 \\
\hline NIRCAM_F480M & 4.8181 & 4.8167 & 4.8094 & 4.8206 & 4.8154 & 4.8314 & 4.8197 & 0.5449 & 0.3073 & 0.3145 & 0.2253 & 29.2044 & $2.08078 \mathrm{E}-12$ & 161.03 \\
\hline MIRI_F560W & 5.6462 & 5.6362 & 5.5880 & 5.6661 & 5.6262 & 5.6138 & 5.6161 & 1.6516 & 0.9980 & 1.1178 & 0.1893 & 29.8562 & $1.14167 \mathrm{E}-12$ & 120.97 \\
\hline MIRI_F770W & 7.6669 & 7.6428 & 7.5260 & 7.7145 & 7.6188 & 7.5950 & 7.5977 & 2.4617 & 1.9647 & 2.1026 & 0.2962 & 31.1385 & 3.50433E-13 & 68.28 \\
\hline MIRI_F1000W & 9.9694 & 9.9544 & 9.8806 & 9.9994 & 9.9394 & 9.9255 & 9.9272 & 2.4395 & 1.7910 & 1.8730 & 0.2867 & 32.2809 & $1.22357 \mathrm{E}-13$ & 40.44 \\
\hline MIRI_F1130W & 11.3111 & 11.3087 & 11.2962 & 11.3161 & 11.3062 & 11.3035 & 11.3050 & 1.5212 & 0.7336 & 0.7128 & 0.1577 & 32.8409 & 7.30515E-14 & 31.16 \\
\hline MIRI_F1500W & 15.0929 & 15.0651 & 14.9272 & 15.1485 & 15.0373 & 15.0153 & 15.0107 & 4.3004 & 2.9217 & 3.1126 & 0.2387 & 34.0608 & 2.37513E-14 & 17.98 \\
\hline MIRI_F1800W & 18.0088 & 17.9865 & 17.8760 & 18.0536 & 17.9641 & 17.9332 & 17.9422 & 4.7688 & 2.9569 & 2.9851 & 0.2070 & 34.8322 & $1.16713 \mathrm{E}-14$ & 12.59 \\
\hline MIRI_F2100W & 20.8425 & 20.7950 & 20.5607 & 20.9372 & 20.7476 & 20.7067 & 20.6991 & 7.1237 & 4.5749 & 4.6813 & 0.1953 & 35.4510 & $6.60100 \mathrm{E}-15$ & 9.52 \\
\hline MIRI_F2550W & 25.4081 & 25.3639 & 25.1519 & 25.4992 & 25.3197 & 25.2881 & 25.2803 & 8.0027 & 3.6615 & 3.4294 & 0.1019 & 36.3163 & $2.97500 \mathrm{E}-15$ & 6.38 \\
\hline NIRISS_F090W & 0.9058 & 0.9031 & 0.8985 & 0.9134 & 0.9004 & 0.9071 & 0.9095 & 0.2404 & 0.1833 & 0.2093 & 0.5295 & 22.6748 & $8.51330 \mathrm{E}-10$ & 2316.26 \\
\hline NIRISS_F115W & 1.1528 & 1.1499 & 1.1388 & 1.1588 & 1.1470 & 1.1490 & 1.1491 & 0.3180 & 0.2499 & 0.2699 & 0.5486 & 23.4581 & 4.13773E-10 & 1824.86 \\
\hline NIRISS_F140M & 1.4054 & 1.4044 & 1.4010 & 1.4075 & 1.4035 & 1.4048 & 1.4034 & 0.2236 & 0.1424 & 0.1481 & 0.4086 & 24.2231 & $2.04526 \mathrm{E}-10$ & 1345.65 \\
\hline NIRISS_F150W & 1.4970 & 1.4936 & 1.4797 & 1.5040 & 1.4902 & 1.4907 & 1.4887 & 0.4145 & 0.3160 & 0.3399 & 0.4856 & 24.4520 & $1.65658 \mathrm{E}-10$ & 1232.74 \\
\hline NIRISS_F158M & 1.5851 & 1.5825 & 1.5704 & 1.5895 & 1.5799 & 1.5766 & 1.5739 & 0.8980 & 0.1990 & 0.2011 & 0.1222 & 24.6817 & $1.34061 \mathrm{E}-10$ & 1119.85 \\
\hline NIRISS_F200W & 1.9979 & 1.9930 & 1.9714 & 2.0077 & 1.9880 & 1.9857 & 1.9865 & 0.5681 & 0.4225 & 0.4741 & 0.5126 & 25.5611 & $5.96423 \mathrm{E}-11$ & 790.19 \\
\hline NIRISS_F277W & 2.7737 & 2.7641 & 2.7110 & 2.7910 & 2.7545 & 2.7242 & 2.7463 & 2.4443 & 0.6915 & 0.7281 & 0.2001 & 26.8736 & $1.78060 \mathrm{E}-11$ & 453.79 \\
\hline NIRISS_F356W & 3.6036 & 3.5926 & 3.5326 & 3.6243 & 3.5817 & 3.4346 & 3.5737 & 1.1841 & 0.9093 & 0.9242 & 0.5690 & 27.9544 & $6.58052 \mathrm{E}-12$ & 283.31 \\
\hline NIRISS_F380M & 3.8258 & 3.8229 & 3.7742 & 3.8284 & 3.8199 & 3.3856 & 3.8125 & 0.4586 & 0.2050 & 0.2056 & 0.3234 & 28.2278 & $5.11523 \mathrm{E}-12$ & 249.36 \\
\hline NIRISS_F430M & 4.2822 & 4.2792 & 4.2303 & 4.2848 & 4.2762 & 4.2904 & 4.2710 & 0.3414 & 0.2016 & 0.2135 & 0.3908 & 28.6952 & $3.32612 \mathrm{E}-12$ & 203.16 \\
\hline NIRISS_F444W & 4.4400 & 4.4270 & 4.3587 & 4.4653 & 4.4139 & 3.9705 & 4.4054 & 1.4206 & 1.0923 & 1.1403 & 0.5291 & 28.8293 & $2.93953 \mathrm{E}-12$ & 192.16 \\
\hline NIRISS_F480M & 4.8147 & 4.8113 & 4.7529 & 4.8181 & 4.8080 & 4.8294 & 4.7985 & 0.5192 & 0.2968 & 0.3026 & 0.3206 & 29.1856 & $2.11727 \mathrm{E}-12$ & 163.49 \\
\hline OMEGACAM_u & 0.3594 & 0.3590 & 0.3632 & 0.3602 & 0.3585 & 0.3242 & 0.3661 & 0.0851 & 0.0461 & 0.0527 & 0.1078 & 21.0647 & 3.75085E-09 & 1612.09 \\
\hline OMEGACAM_g & 0.4751 & 0.4735 & 0.4702 & 0.4783 & 0.4719 & 0.3788 & 0.4771 & 0.1818 & 0.1150 & 0.1317 & 0.3397 & 20.6584 & 5.45315E-09 & 4077.99 \\
\hline OMEGACAM_r & 0.6289 & 0.6276 & 0.6233 & 0.6316 & 0.6263 & 0.6260 & 0.6264 & 0.1963 & 0.1275 & 0.1351 & 0.3237 & 21.5293 & 2.44507E-09 & 3212.63 \\
\hline OMEGACAM_i & 0.7508 & 0.7495 & 0.7453 & 0.7535 & 0.7482 & 0.7504 & 0.7491 & 0.2451 & 0.1143 & 0.1258 & 0.1920 & 22.1133 & $1.42790 \mathrm{E}-09$ & 2675.72 \\
\hline OMEGACAM_z & 0.8847 & 0.8842 & 0.8840 & 0.8856 & 0.8837 & 0.8822 & 0.8833 & 0.1677 & 0.0606 & 0.0530 & 0.0945 & 22.6336 & $8.84201 \mathrm{E}-10$ & 2305.75 \\
\hline VIRCAM_Z & 0.8950 & 0.8899 & 0.8815 & 0.9252 & 0.8849 & 0.8903 & 0.8975 & 1.4202 & 0.0929 & 0.0973 & 0.0477 & 22.6456 & $8.74504 \mathrm{E}-10$ & 2310.18 \\
\hline VIRCAM_Y & 1.0274 & 1.0253 & 1.0204 & 1.0363 & 1.0232 & 1.0250 & 1.0287 & 0.3265 & 0.0905 & 0.0924 & 0.1933 & 23.0396 & $6.08351 \mathrm{E}-10$ & 2133.31 \\
\hline VIRCAM_H & 1.2549 & 1.2535 & 1.2480 & 1.2586 & 1.2520 & 1.2502 & 1.2539 & 0.2619 & 0.1624 & 0.1720 & 0.4769 & 23.7910 & $3.04518 \mathrm{E}-10$ & 1595.92 \\
\hline VIRCAM_J & 1.6453 & 1.6430 & 1.6339 & 1.6499 & 1.6407 & 1.6374 & 1.6373 & 0.4530 & 0.2797 & 0.2905 & 0.5340 & 24.8212 & $1.17897 \mathrm{E}-10$ & 1061.59 \\
\hline VIRCAM_Ks & 2.1521 & 2.1494 & 2.1349 & 2.1567 & 2.1468 & 2.1840 & 2.1417 & 1.3215 & 0.2894 & 0.3078 & 0.1869 & 25.8668 & $4.50069 \mathrm{E}-11$ & 693.60 \\
\hline SkyMapper_u & 0.3616 & 0.3590 & 0.3685 & 0.3696 & 0.3565 & 0.3475 & 0.3267 & 0.4341 & 0.0456 & 0.0431 & 0.1040 & 21.1729 & 3.39505E-09 & 1459.58 \\
\hline SkyMapper_v & 0.3837 & 0.3836 & 0.3874 & 0.3841 & 0.3834 & 0.3817 & 0.3831 & 0.0649 & 0.0318 & 0.0310 & 0.4905 & 20.6070 & $5.71726 \mathrm{E}-09$ & 2805.75 \\
\hline SkyMapper_g & 0.5099 & 0.5075 & 0.5016 & 0.5148 & 0.5051 & 0.5088 & 0.5044 & 0.2595 & 0.1477 & 0.1570 & 0.5693 & 20.8530 & 4.55807E-09 & 3916.05 \\
\hline SkyMapper_r & 0.6157 & 0.6138 & 0.6078 & 0.6195 & 0.6120 & 0.6131 & 0.6134 & 0.2395 & 0.1524 & 0.1582 & 0.6359 & 21.4523 & $2.62480 \mathrm{E}-09$ & 3298.97 \\
\hline SkyMapper_i & 0.7778 & 0.7768 & 0.7734 & 0.7799 & 0.7758 & 0.7757 & 0.7750 & 0.1896 & 0.1202 & 0.1400 & 0.6336 & 22.2365 & $1.27465 \mathrm{E}-09$ & 2565.56 \\
\hline SkyMapper_z & 0.9159 & 0.9143 & 0.9119 & 0.9191 & 0.9128 & 0.9271 & 0.9206 & 0.2445 & 0.1110 & 0.0849 & 0.4540 & 22.7153 & $8.20138 \mathrm{E}-10$ & 2286.99 \\
\hline
\end{tabular}




\section{ORCID iDs}

Christopher N. A. Willmer (1) https://orcid.org/0000-00019262-9997

\section{References}

Aumann, H. H., Beichman, C. A., Gillett, F. C., et al. 1984, ApJL, 278, L23 Beers, T. C., Flynn, K., \& Gebhardt, K. 1990, AJ, 100, 32

Bessell, M., Bloxham, G., Schmidt, B., et al. 2011, PASP, 123, 789

Bessell, M., \& Murphy, S. 2012, PASP, 124, 140, (BM12)

Bessell, M. S., \& Brett, J. M. 1988, PASP, 100, 1134

Bessell, M. S., Castelli, F., \& Plez, B. 1998, A\&A, 333, 231

Binney, J., \& Merrifield, M. 1998, Galactic Astronomy (Princeton, NJ: Princeton Univ. Press), 53

Blanton, M. R., Hogg, D. W., Bahcall, N. A., et al. 2003, ApJ, 592, 819

Bohlin, R. C. 2014, AJ, 147, 127

Bohlin, R. C., Gordon, K. D., \& Tremblay, P.-E. 2014, PASP, 126, 711

Bohlin, R. C., Mészáros, S., Fleming, S. W., et al. 2017, AJ, 153, 234

Budding, E. 1993, Introduction to Astronomical Photometry (Cambridge: Cambridge Univ. Press), 286

Carter, B. S. 1990, MNRAS, 242, 1

Casagrande, L., Ramírez, I., Meléndez, J., \& Asplund, M. 2012, ApJ, 761, 16

Cohen, M., Walker, R. G., Barlow, M. J., \& Deacon, J. R. 1992, AJ, 104, 1650

Cohen, M., Wheaton, W. A., \& Megeath, S. T. 2003, AJ, 126, 1090

Cousins, A. W. J. 1976, MmRAS, 81, 25

Dudok de Wit, T., Kopp, G., Fröhlich, C., \& Schöll, M. 2017, GeoRL, 44, 1196

Engelke, C. W. 1992, AJ, 104, 1248

Engelke, C. W., Price, S. D., \& Kraemer, K. E. 2010, AJ, 140, 1919

Falcón-Barroso, J., Sánchez-Blázquez, P., Vazdekis, A., et al. 2011, A\&A, 532, A95

Fontenla, J. M., Harder, J., Livingston, W., Snow, M., \& Woods, T. 2011, JGRD, 116, D20108

Fukugita, M., Shimasaku, K., \& Ichikawa, T. 1995, PASP, 107, 945

Gillett, F. C., Clegg, P., Rosing, D., et al. 1984, IRAS Explanatory Supplement (Pasadena, CA: IPAC), http://irsa.ipac.caltech.edu/IRASdocs/exp.sup/ toc.html

Glasse, A. \& MIRI European Consortium 2015, MIRI-TN-00072-ATC issue 3

Goddard Space Flight Center 2012, Galaxy Evolution Explorer (Greenbelt, MD: GSFC), https://universe.gsfc.nasa.gov/archive/galex/
Gunn, J. E., Carr, M., Rockosi, C., et al. 1998, AJ, 116, 3040

Gwyn, S. D. J. 2012, AJ, 143, 38

Haberreiter, M., Schöll, M., Dudok de Wit, T., et al. 2017, JGRA, 122 , 5910

Hewett, P. C., Warren, S. J., Leggett, S. K., \& Hodgkin, S. T. 2006, MNRAS, 367,454

Høg, E., Fabricius, C., Makarov, V. V., et al. 2000, A\&A, 355, L27

Jarrett, T. H., Cohen, M., Masci, F., et al. 2011, ApJ, 735, 112

Johnson, H. L. 1955, AnAp, 18, 292

Johnson, H. L. 1966, ARA\&A, 4, 193

Johnson, H. L., \& Morgan, W. W. 1953, ApJ, 117, 313

Kurucz, R. L. 2005, http://kurucz.harvard.edu

Kurucz, R. L. 2011, http://kurucz.harvard.edu/stars/Sun/fsunallp.500resam501

Maíz Apellániz, J. 2006, AJ, 131, 1184

Mann, A. W., \& von Braun, K. 2015, PASP, 127, 102

NASA/IPAC Infrared Science Archive 2008, Spitzer Documentation \& Tools (Pasadena, CA: IRSA), http://irsa.ipac.caltech.edu/data/SPITZER/docs/ spitzermission/

National Optical Astronomy Observatories 2015, The NOAO Data Handbook (Tucson, AZ: NOAO), http://ast.noao.edu/data/docs

Oke, J. B., \& Gunn, J. E. 1983, ApJ, 266, 713

Peterson, D. M., Hummel, C. A., Pauls, T. A., et al. 2006, Natur, 440, 896

Prša, A., Harmanec, P., Torres, G., et al. 2016, AJ, 152, 41

Ramírez, I., Michel, R., Sefako, R., et al. 2012, ApJ, 752, 5

Reach, W. T., Megeath, S. T., Cohen, M., et al. 2005, PASP, 117, 978

Rieke, G. H., Blaylock, M., Decin, L., et al. 2008, AJ, 135, 2245

Space Telescope Science Institute 1998, SYNPHOT Users Guide (Baltimore, MD: STScI), http://stsdas.stsci.edu/Files/SynphotManual.pdf

Space Telescope Science Institute 2017a, CALSPEC Calibration Database (Baltimore, MD: STScI), http://www.stsci.edu/hst/observatory/cdbs/ calspec.html

Space Telescope Science Institute 2017b, JWST Observatory and Instrumentation Documentation (Baltimore, MD: STScI), https://jwstdocs.stsci.edu/ display/JTI/

Su, K. Y. L., Rieke, G. H., Malhotra, R., et al. 2013, ApJ, 763, 118

Thuillier, G., Floyd, L., Woods, T. N., et al. 2004, AdSpR, 34, 256

Thuillier, G., Hersé, M., Labs, D., et al. 2003, SoPh, 214, 1

Tokunaga, A. T., \& Vacca, W. D. 2005, PASP, 117, 421

Tonry, J. L., Stubbs, C. W., Lykke, K. R., et al. 2012, ApJ, 750, 99

Woods, T. N., Chamberlin, P. C., Harder, J. W., et al. 2009, GeoRL, 36 L01101 